\title{
The Tip-Link Antigen, a Protein Associated with the Transduction Complex of Sensory Hair Cells, Is Protocadherin-15
}

\author{
Zubair M. Ahmed, ${ }^{\ddagger}{ }^{\ddagger}$ Richard Goodyear,${ }^{2 \ddagger}$ Saima Riazuddin,${ }^{1 *}$ Ayala Lagziel,${ }^{1 \star}$ P. Kevin Legan, ${ }^{2}$ Martine Behra,${ }^{3}$ \\ Shawn M. Burgess, ${ }^{3}$ Kathryn S. Lilley, ${ }^{4}$ Edward R. Wilcox, ${ }^{5}$ Sheikh Riazuddin, ${ }^{6}$ Andrew J. Griffith, ${ }^{1}$ \\ Gregory I. Frolenkov, ${ }^{7}$ Inna A. Belyantseva, ${ }^{1}$ Guy P. Richardson, ${ }^{2}$ and Thomas B. Friedman ${ }^{1}$ \\ ${ }^{1}$ Laboratory of Molecular Genetics, National Institute on Deafness and Other Communication Disorders, National Institutes of Health, Rockville, Maryland \\ 20850, ${ }^{2}$ School of Life Sciences, University of Sussex, Falmer, Brighton BN1 9QG, United Kingdom, ${ }^{3}$ Genome Technology Branch, National Human Genome \\ Research Institute, National Institutes of Health, Bethesda, Maryland 20892, ${ }^{4}$ Cambridge Center for Proteomics, Department of Biochemistry, University of \\ Cambridge, Cambridge CB2 1QW, United Kingdom, ${ }^{5}$ DNA Sequencing Center, Department of Integrative Biology, Brigham Young University, Provo, Utah \\ 84602, ${ }^{6}$ National Centre of Excellence in Molecular Biology, Lahore, Pakistan, and ${ }^{7}$ Department of Physiology, University of Kentucky, Lexington, Kentucky \\ 40536
}

Sound and acceleration are detected by hair bundles, mechanosensory structures located at the apical pole of hair cells in the inner ear. The different elements of the hair bundle, the stereocilia and a kinocilium, are interconnected by a variety of link types. One of these links, the tip link, connects the top of a shorter stereocilium with the lateral membrane of an adjacent taller stereocilium and may gate the mechanotransducer channel of the hair cell. Mass spectrometric and Western blot analyses identify the tip-link antigen, a hitherto unidentified antigen specifically associated with the tip and kinocilial links of sensory hair bundles in the inner ear and the ciliary calyx of photoreceptors in the eye, as an avian ortholog of human protocadherin-15, a product of the gene for the deaf/blindness Usher syndrome type 1F/DFNB23 locus. Multiple protocadherin-15 transcripts are shown to be expressed in the mouse inner ear, and these define four major isoform classes, two with entirely novel, previously unidentified cytoplasmic domains. Antibodies to the three cytoplasmic domain-containing isoform classes reveal that each has a different spatiotemporal expression pattern in the developing and mature inner ear. Two isoforms are distributed in a manner compatible for association with the tip-link complex. An isoform located at the tips of stereocilia is sensitive to calcium chelation and proteolysis with subtilisin and reappears at the tips of stereocilia as transduction recovers after the removal of calcium chelators. Protocadherin- 15 is therefore associated with the tip-link complex and may be an integral component of this structure and/or required for its formation.

Key words: protocadherin-15; tip link; TLA; stereocilia; mechanotransduction; hair cell

\section{Introduction}

Hearing and balance depend on hair cells, polarized epithelial cells of the inner ear that have a mechanosensitive hair bundle located at their apical pole. The hair bundle is composed of nu-

\footnotetext{
Received March 17, 2006; revised May 18, 2006; accepted May 19, 2006.

This work was supported by National Institute on Deafness and Other Communication Disorders-National Institutes of Health Intramural Funds 1 Z01 DC000039-09 LMG (T.B.F.) and by The Wellcome Trust Grant 071394/Z/03/Z (G.P.R.). We thank Richard Chadwick, Penelope Friedman, Dennis Drayna, Robert Morell, Julie Schultz, and Doris Wu for their critiques of this manuscript. We are indebted to Rong-Fong Shen and Guanghui Wang (Proteomics Core, National Heart, Lung, and Blood Institute-National Institutes of Health) for their suggestions and analyses of mass spectrometry data.

${ }^{\ddagger}$ Z.M.A. and R.G. are co-first authors.

*S.R. and A.L. contributed equally to this work.

Correspondence should be addressed to either of the following: Dr. Guy P. Richardson, School of Life Sciences, University of Sussex, Falmer, Brighton, BN1 9QG, UK, E-mail: g.p.richardson@sussex.ac.uk; or Dr. Thomas B. Friedman, Section on Human Genetics, Laboratory of Molecular Genetics, National Institute on Deafness and Other Communication Disorders, National Institutes of Health, 5 Research Court, Rockville, MD 20850, E-mail: friedman@nidcd.nih.gov.

DOI:10.1523/JNEUROSCI.1163-06.2006

Copyright $\odot 2006$ Society for Neuroscience $\quad 0270-6474 / 06 / 267022-13 \$ 15.00 / 0$
}

merous stereocilia and, in all organs except the mature cochlea, a single kinocilium. These elements are coupled to one another by a variety of links. Up to four distinct interstereocilial link types, tip links, horizontal top connectors, shaft connectors, and ankle links, can be distinguished in avian hair bundles (Goodyear and Richardson, 1992). A fifth link type, the kinocilial link, connects the kinocilium, when present, to the adjacent stereocilia (Goodyear and Richardson, 2003). The tip link, a slender filament connecting the top of one stereocilium to the side of an adjacent taller stereocilium, is a feature of all hair bundles and is thought to gate the mechanotransducer channel of the hair cell (Pickles et al., 1984; Assad et al., 1991). The other links, which can vary in their distribution according to species, age, and hair-bundle type, may hold stereocilia together as a coherent unit and transmit forces across the bundle.

Distinct antigens detected by monoclonal antibodies (mAbs) are associated with three of the four interstereocilial links found in the chick inner ear. The hair-cell antigen (HCA) is associated with shaft connectors (Goodyear and Richardson, 1992), the 
ankle-link antigen (ALA) with ankle links (Goodyear and Richardson, 1999), and the tip-link antigen (TLA) with tip and kinocilial links (Goodyear and Richardson, 2003). Some of these antigens and other candidate link components have been recently identified. Cadherin-23 is a component of the kinocilial links and transient lateral links of immature hair bundles (Lagziel et al., 2005; Michel et al., 2005) and a candidate for a component of the tip link in mature hair cells (Siemens et al., 2004). The HCA is the receptor-like inositol lipid phosphatase Ptprq (Goodyear et al., 2003), and the ALA is the very large G-protein-coupled receptor Vlgr1 (Goodyear et al., 2006). Usherin and protocadherin-15 (Ahmed et al., 2001; Alagramam et al., 2001a) may also be associated with ankle links (Adato et al., 2005; Senften et al., 2006). Protocadherin-15 is a member of the cadherin superfamily of $\mathrm{Ca}^{2+}$-dependent adhesion glycoproteins, and its importance for the normal architecture of the hair cell stereocilia bundles and inner ear function is well established in humans, mice, and zebrafish (Ahmed et al., 2001, 2003; Alagramam et al., 2001a,b; Ben-Yosef et al., 2003; Seiler et al., 2005).

In this study, we identify the TLA by mass spectrometry peptide sequencing as an epitope of protocadherin-15. Furthermore, we reveal that novel isoform classes of protocadherin-15 are expressed in the mouse and chicken inner ear and that these can be defined by the absence or presence of one of three alternative unique cytoplasmic domains. Three of these isoform classes are shown to have dramatically different spatiotemporal expression patterns within the developing and mature hair bundle, and two are distributed in a manner that allows association with the tiplink complex.

\section{Materials and Methods}

cDNA cloning and sequence analysis. Full-length mouse $P c d h 15$ poly $(\mathrm{A})^{+}$ RNA was isolated from postnatal day $1(\mathrm{P} 1)$ to $\mathrm{P} 5$ inner ear tissue dissected from 50 C57BL/6J mice using Poly(A)Pure (Ambion, Austin, TX). cDNA was prepared using an oligo-dT primer and PowerScript reverse transcriptase (Clontech, Cambridge, UK). PCDH15 transcripts were amplified from human retina cDNA (GETRare; Genemed Synthesis, South San Francisco, CA). To determine the structure and isoforms of chicken $P c d h 15$, we used poly $\left(\mathrm{A}^{+}\right)$RNA from chicken brain (Stratagene, La Jolla, CA). Supplemental Table S1 (available at www.jneurosci.org as supplemental material) provides the sequences of the primers used to amplify PCDH15/Pcdh15 from human, mouse, and chicken tissues. All PCR products were subcloned, and both strands were fully sequenced.

Antibodies. Mouse mAb G19 directed against the TLA from chicken inner ear hair cells, mAb D10 directed against the avian hair-cell antigen, and antisera to protocadherin-15-CD1 (PB303) were characterized and validated as reported previously (Richardson et al., 1990; Ahmed et al., 2003; Goodyear and Richardson, 2003). Additional peptides based on mouse protocadherin-15 (shown in Fig. 1) were synthesized by Princeton BioMolecules (Langhorn, PA) and used to immunize New Zealand white rabbits (Covance Research Products, Denver, PA). The sequences of peptide immunogens are listed in supplemental Table S2 (available at www.jneurosci.org as supplemental material). Antisera HL5383 is directed against an expressed fusion protein corresponding to the full length of the unique sequence (exon 39) of the CD3 cytoplasmic domain (see Fig. $1 c$, pink region) and prepared by cloning cDNAs encoding residues 1516-1649 (supplemental Tables S2, S3, available at www. jneurosci.org as supplemental material). Antisera HL5614 is directed against an expressed fusion protein corresponding to sequence from the $\mathrm{N}$ terminus of mouse protocadherin-15 (residues 28-335; GenBank accession number AAG53891). Both cDNAs were cloned into pGEX5.1 (Amersham Biosciences, Arlington Heights, IL), expressed in Escherichia coli (BL21Gold DE3 pLysS; Stratagene), purified as described previously (Belyantseva et al., 2005), and injected into rabbits (Covance Research Products). To affinity purify antisera HL5383 and HL5614, the sequences encoding the same amino acid residues, $1516-1649$ and $28-335$, respec- tively, were cloned into pMAL-c2x (New England Biolabs, Beverly, MA), transformed into E. coli Rosetta DE3 (Novagen, Madison, WI), and purified on amylose resin (Lagziel et al., 2005). A column of MBPprotocadherin-15-CD3 (residues 1516-1649) and MBP-protocadherin-15-Nter bound to 4\% beaded agarose (Amino-Link Plus) were then used to affinity purify rabbit antisera HL5383 raised against glutathione $S$-transferase (GST)-CD3 and HL5614 against GST-Nter.

To prepare an antiserum to the cytoplasmic domain of the chicken protocadherin-15-CD1, a fragment encoding the C-terminal 194 amino acids of chicken protocadherin-15-CD1 was amplified by reverse transcription (RT)-PCR with Pfu polymerase (Stratagene) and primers GgPcdh15F2 (gcagccatatgCTTTCTCCTCCACCAACTC) and GgPcdh15R1 (cagccggatcctCACAGTGCTGTCGACTGAGATG) from total RNA isolated from $2 \mathrm{~d}$ posthatch chick utricle. The PCR product was digested with $\mathrm{NdeI}$ and $\mathrm{BamHI}$ and ligated into the same sites of pET15b (Novagen, Nottingham, UK). The 6His-tagged fusion protein was expressed in E. coli BL21 (DE3) pLysS and purified by nickel affinity column chromatography. The purified fusion protein was used to immunize a CD1 mouse, and the immune serum obtained from a tail bleed was used at a dilution of 1:1000 for immunoblotting.

The specificity of antibodies raised against different epitopes of protocadherin- 15 was validated using inner ear tissue from homozygous $a v-3 J$ mice and by blocking assays performed with transiently transfected cell lines. A Flag-tagged sequence encoding the CD3 cytoplasmic domain (residues 1420-1682; GenBank accession number DQ354413) (supplemental Table S3, available at www.jneurosci.org as supplemental material) was used to validate antisera PB375 and HL5383, whereas a Flagtagged CD2 cytoplasmic domain was used to evaluate antisera PB464-2B (residues 1555-1790; GenBank accession number DQ354405). A Histagged construct of protocadherin-15-CD1 [sequence encoding the N terminus and the first six extracellular (EC) domains (residues 1-900), a His-epitope tag, and residues 1410-1943 encoding the transmembrane domain and CD1 (GenBank accession number AAG53891] was used to validate antisera PB303, PB473-3, and HL5614. All epitope-tagged constructs were cloned into expression vector pcDNA3.1 (Invitrogen, Carlsbad, CA) and transfected (Lipofectamine 2000; Invitrogen) into HeLa or human lymphoblastoid cells. After incubation for $24 \mathrm{~h}$ at $37^{\circ} \mathrm{C}\left(5 \% \mathrm{CO}_{2}\right)$ in DMEM supplemented with 10\% FCS (Invitrogen), cells were fixed with $4 \%$ paraformaldehyde in PBS $(1 \mathrm{~h})$, permeabilized in $0.5 \%$ Triton X-100 (TX-100) for $10 \mathrm{~min}$, and washed in PBS. Nonspecific binding sites were blocked using NGS-BSA [5\% normal goat serum (Invitrogen) and $2 \%$ bovine serum albumin (MP Biomedicals, Irvine, CA)] in PBS. Depending on the expression construct, cells were incubated for $2 \mathrm{~h}$ in the appropriate anti-protocadherin-15 antisera (PB303, PB375, PB4642B, PB473-3, HL5383, or HL5614) at a concentration of $\sim 5 \mu \mathrm{g} / \mathrm{ml}$ in NGS-BSA.

For antisera blocking experiments, anti-protocadherin-15 antisera were preabsorbed for 30 min with a 10-fold excess concentration (over that of affinity-purified antisera) of the corresponding expressed protein or peptide(s) and then incubated with transfected cells for $2 \mathrm{~h}$. Cells were rinsed three times in PBS and were incubated with anti-Flag (Roche Products, Welwyn Garden City, CA) or anti-His (Roche Products) monoclonal antibodies for $2 \mathrm{~h}$. After three rinses in PBS, samples were incubated in a 1:200 dilution of the FITC-conjugated anti-rabbit IgG secondary antibody (Amersham Biosciences) and 1:200 dilution of the Alexa 568-conjugated anti-mouse IgG secondary antibody (Invitrogen) for $30 \mathrm{~min}$. Samples were washed three times with PBS and mounted using a ProLong Antifade kit (Invitrogen) and viewed in an LSM510 Zeiss (Oberkochen, Germany) confocal microscope. There are five identical amino acid residues (DYLRL) in the cytoplasmic domains of cadherin 23 and protocadherin-15-CD2. We tested our protocadherin-15 antibodies for cross-reactivity with cadherin-23 and found none.

Immunocytochemistry. Immunocytochemistry was performed as described previously (Belyantseva et al., 2005; Michel et al., 2005). For labeling experiments involving cochlear cultures and whole mounts, 4 to 12 explants or maculae were examined for each condition.

Immunoaffinity purification of TLA. mAb G19 recognizes an extracellular epitope associated with the tip link (Goodyear and Richardson, 2003). An affinity column was prepared commercially (Immune Sys- 
tems, Bristol, UK) by conjugating $11 \mathrm{mg}$ of mAb G19 that had been affinity purified from hybridoma supernatant on Protein A to $3 \mathrm{ml}$ of Sepharose CL-4B resin. Retinas were dissected from 180 2-d posthatch chicken eyes in PBS containing protease inhibitors (1 mM PMSF, $2 \mathrm{~mm}$ benzamidine, $1 \mu \mathrm{g} / \mathrm{ml}$ leupeptin, and $1 \mu \mathrm{g} / \mathrm{ml}$ pepstatin) and frozen. The frozen retinas were thawed and homogenized in $50 \mathrm{ml}$ of extraction buffer (150 mм NaCl, 1\% TX-100, $5 \mathrm{~mm} \mathrm{CaCl}_{2}$, and $20 \mathrm{~mm} \mathrm{HEPES,} \mathrm{pH}$ 7.2) containing EDTA-free protease inhibitor cocktail (Roche Products) and centrifuged at $41,000 \times g_{\max }$ for $30 \mathrm{~min}$. The resultant supernatant was filtered through Whatman (Maidstone, UK) \#1 filter paper, recentrifuged at $48,000 \times g_{\max }$ for $30 \mathrm{~min}$, and passed three times through the
mAb G19 affinity column. The column was washed sequentially with 40 $\mathrm{ml}$ of extraction buffer, $60 \mathrm{ml}$ of high-salt wash buffer $(0.5 \mathrm{M} \mathrm{NaCl}, 0.1 \%$ TX-100, $5 \mathrm{~mm} \mathrm{CaCl}_{2}$, and $20 \mathrm{~mm}$ HEPES, pH 7.2), and $10 \mathrm{ml}$ of $1 \mathrm{M} \mathrm{MgCl}_{2}$ (in $0.1 \% \mathrm{TX}-100,5 \mathrm{~mm} \mathrm{CaCl}_{2}$, and $20 \mathrm{~mm}$ HEPES, $\mathrm{pH}$ 7.2), and finally eluted with $6 \mathrm{ml}$ of glycine- $\mathrm{HCl}, \mathrm{pH} 2.0$. The glycine- $\mathrm{HCl}$ eluate was neutralized with Tris base, dialyzed extensively against several changes of water, and lyophilized. The lyophilized sample was resuspended in reducing SDS-PAGE sample buffer, heated at $100^{\circ} \mathrm{C}$ for 4 min, and separated on a $6 \%$ polyacrylamide gel. Coomassie-stained protein bands of $\sim 250$ and $200 \mathrm{kDa}$ corresponding to the previously identified TLA were excised and washed extensively in water.

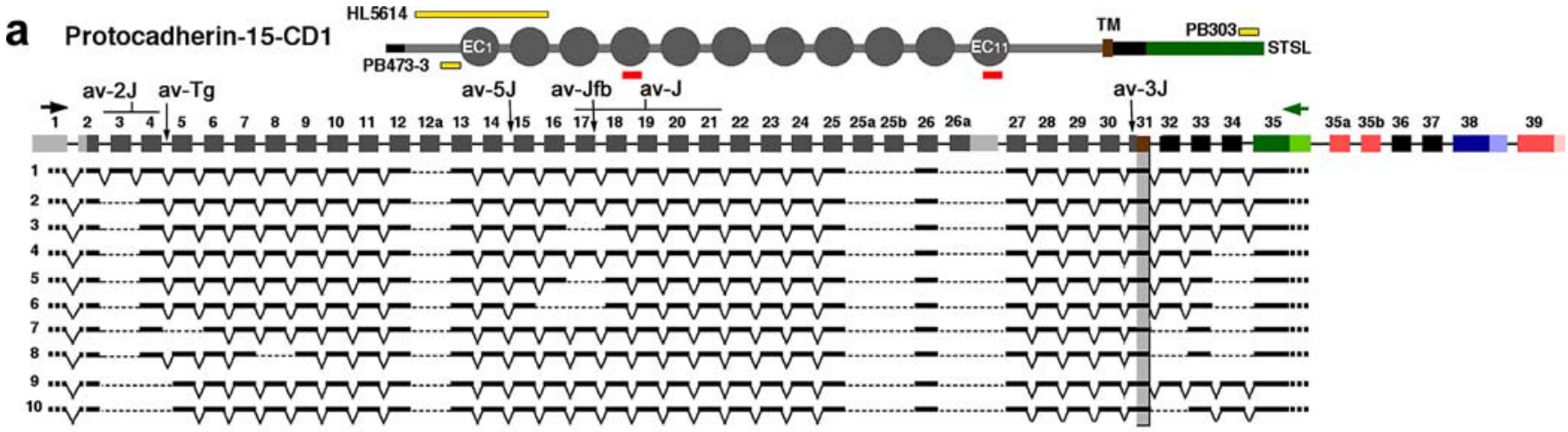

b Protocadherin-15-CD2

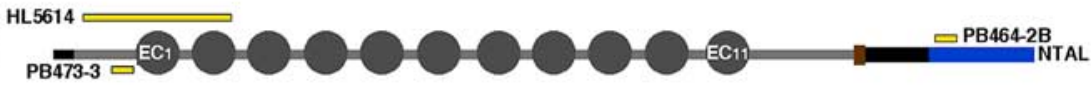

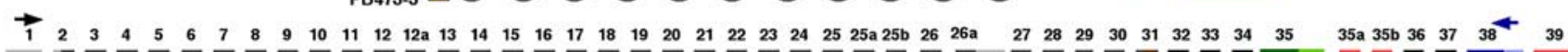

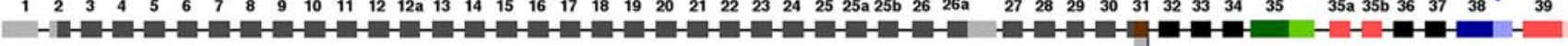
(n)

\section{Protocadherin-15-CD3}

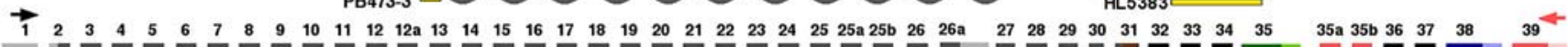
1 …ㄱำ

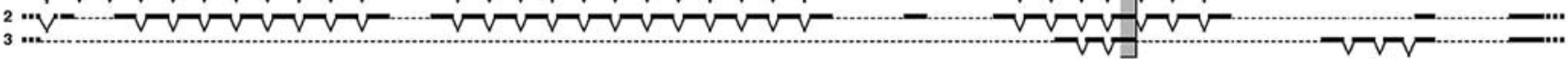

\section{d Protocadherin-15-SI}

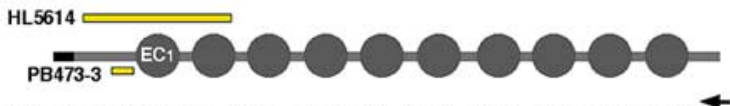

$\begin{array}{llllllllllllllllllllllllllllll}1 & 2 & 3 & 4 & 5 & 6 & 7 & 8 & 9 & 10 & 11 & 12 & 12 a & 13 & 14 & 15 & 16 & 17 & 18 & 19 & 20 & 21 & 22 & 23 & 24 & 25 & 25 a & 25 b & 26 & 26 a\end{array}$

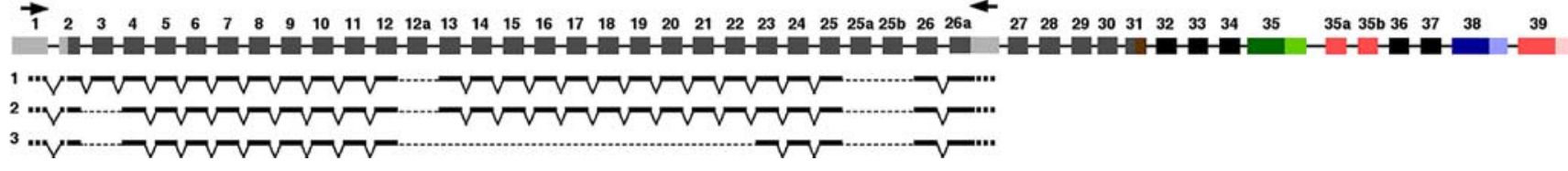

Figure 1. Pcdh15 splice variants. Splicing of the primary transcripts of $P c d h 15$ and the four isoform classes defined by the presence or absence of one of three different cytoplasmic domains. Newly discovered exons of $P$ cdh15 are designated with a letter suffix if located among the reported 35 exons. A dashed line indicates that one or more exons were not included in the transcript. A dotted line designates either the 3' untranslated region (UTR) or the 5' UTR. A signal peptide is encoded by exon 2, and a transmembrane domain (TM; brown) is encoded by exon 31 . $\boldsymbol{a}-\boldsymbol{c}$, Diagrams of exon content of full-length, open reading frame-containing transcripts with one of the three alternative cytoplasmic domains encoded by exons 35 (green), 38 (blue), and 39 (pink), respectively (supplemental Fig. S1, available at www.jneurosci.org as supplemental material). Different antigens used for producing polyclonal antibodies to protocadherin- 15 are shown as yellow rectangles above and below the structures of each protocadherin- 15 isoform. The location of the two tryptic chicken peptides identical to human and mouse sequence that were detected by mass spectrometric analyses of purified TLA are shown as two red rectangles below the structure of protocadherin-15-CD1 (for sequence of peptides, see Table 1). The locations of the six reported Ames waltzer alleles (av-2J, av-Tg2742Rpw, av-5J, av-Jfb, av-J, and av-3J) are shown in $\boldsymbol{a}$. Note that, for all transcripts of mouse Pcdh15 shown in $\boldsymbol{a}-\boldsymbol{c}$, there are no constant coding exons. However, within each class of $P$ cdh 15 transcripts (CD1, CD2, and CD3), the 3' UTR encoded by exons 35, 38, and 39, respectively, appear to be constant, but that observation may reflect an ascertainment bias because the reverse primer for each of the three classes was located in the 3' UTRs (green, blue, and pink arrows) (see also Ahmed et al., 2003). Black arrow in exon 1 is the location of the forward primer. $\boldsymbol{d}$, An isoform class of protocadherin-15 expressed in the mouse inner ear that is predicted to be secreted. The GenBank accession number for each isoform is in supplemental Table S3 (available at www. jneurosci.org as supplemental material). 


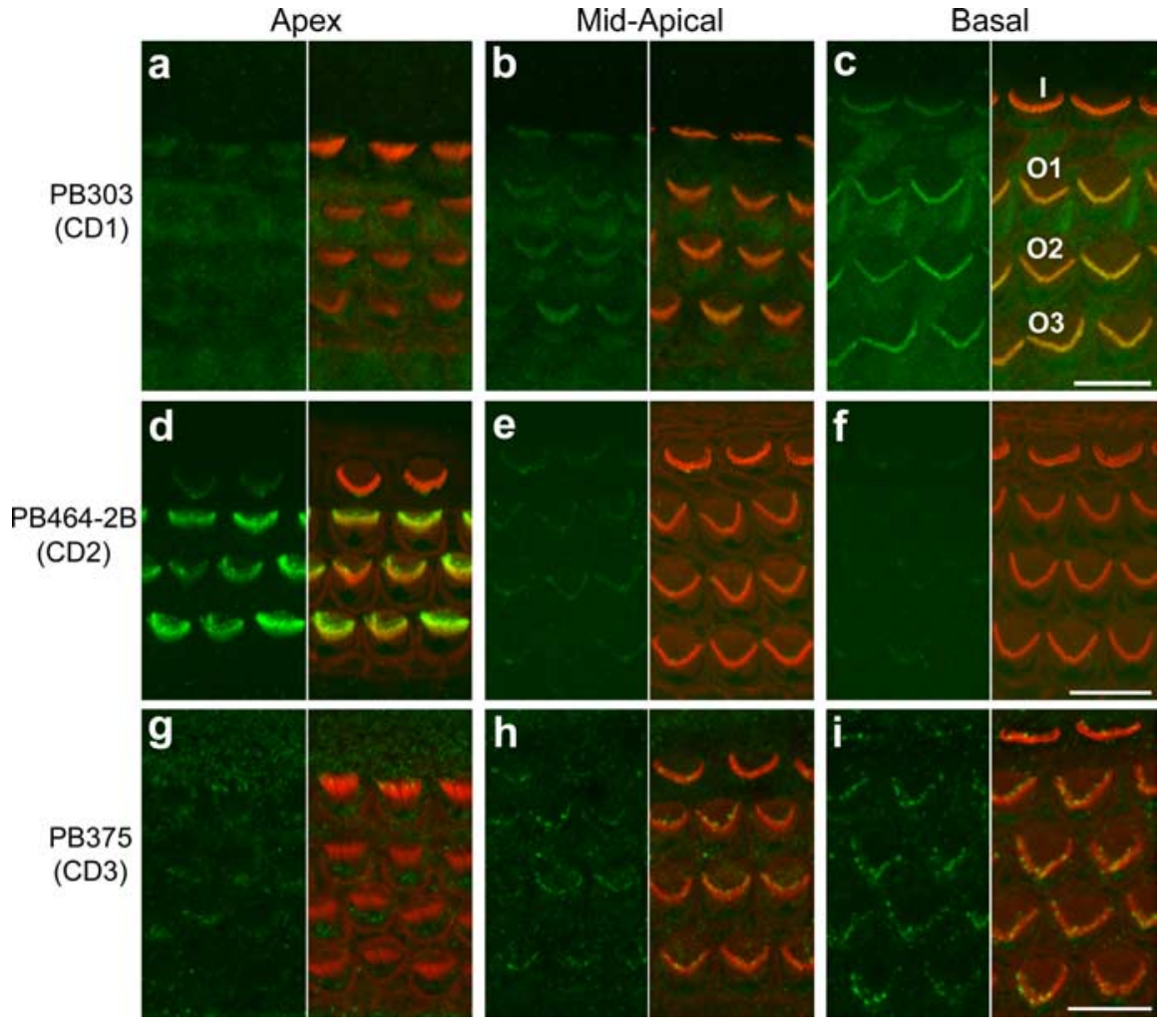

Figure 2. Distributions of protocadherin-15-CD1, protocadherin-15-CD2, and protocadherin-15-CD3 in the developing mouse cochlea. P2 mouse organ of Corti, $1 \mathrm{~d}$ in vitro. Hair bundles from the apical end of the apical coil $(\boldsymbol{a}, \boldsymbol{d}, \boldsymbol{g})$, middle of the apical coil $(\boldsymbol{b}, \boldsymbol{e}, \boldsymbol{h})$, and basal $(\boldsymbol{c}, \boldsymbol{f}, \boldsymbol{i})$ regions of the cochlea stained with antibody PB303 to protocadherin-15-CD1 ( $\boldsymbol{a}-\boldsymbol{c})$, antibody PB464-2B to protocadherin-15-CD2 (d-f), and antibody PB375 to protocadherin-15-CD3 $(\boldsymbol{g}-\boldsymbol{i})$. The left half of each panel shows the distribution of protocadherin-15, and the right half shows the merge with F-actin. I, Inner hair cell; $01,02,03$, outer hair cells in rows 1,2 , and 3 respectively. Scale bars, $10 \mu \mathrm{m}$.

Mass spectrometric analysis of TLA. Acrylamide gel slices containing TLA were reduced, carboxyamidomethylated, digested to peptides using trypsin on a MassPrepStation (Waters, Manchester, UK), desalted, and concentrated. To obtain peptide mass and sequence, the resulting peptides were applied to a capillary liquid chromatography (LC) column coupled to a quadrupole tandem time-of-flight mass spectrometer (LCMS/MS) (QTof2; Waters). For LC-MS/MS, a reverse-phase liquid chromatographic separation of the peptides was accomplished using a PepMap C18 reverse phase column $(75 \mathrm{~mm}$ inner diameter, $15 \mathrm{~cm}$ column; LC Packings, Sunnyvale, CA) on a capillary liquid chromatography system (Waters) attached to the QTof2 mass spectrometer. The MS/MS data obtained from the two TLA protein bands (see Fig. $7 a$, bands 1,2) was then used to search the National Center for Biotechnology Information (NCBI) human and mouse databases using the MASCOT search engine. Probability-based MASCOT scores were used to evaluate identifications. Only matches with $p<0.05$ for random occurrence were considered significant. Because the TLA was affinity purified from the chicken, the same data files were submitted to the search software BioWorks (Thermo Electron Corporation, Waltham, MA). The chicken protein database was downloaded directly from the NCBI, to which chicken protocadherin$15-\mathrm{CD} 1$, protocadherin-15-CD3, and protocadherin-15-CD2/CD3 amino acid sequences were added by us and are now available in the chicken database (supplemental Table S3, available at www.jneurosci.org as supplemental material).

Immunoblot analysis. The TLA was immunoprecipitated from retinal extracts with minor modifications of the method described previously (Goodyear and Richardson, 2003). Retinas were homogenized in extraction buffer containing $1 \%$ Triton X-100 and the protease inhibitors PMSF ( $1 \mathrm{~mm})$, benzamidine $(2 \mathrm{~mm})$, pepstatin $(1 \mu \mathrm{g} / \mathrm{ml})$, and leupeptin $(1 \mu \mathrm{g} / \mathrm{ml})$, centrifuged at $20,000 \times g_{\max }$ for $20 \mathrm{~min}$ at $4^{\circ} \mathrm{C}$. The supernatant was recentrifuged at $4000 \times g_{\max }$ for $10 \mathrm{~min}$, divided into two aliquots, and mixed overnight at $4^{\circ} \mathrm{C}$ with goat anti-mouse $\mathrm{IgG}_{1} \mathrm{Fc}$ agarose beads that had been preloaded with either mAb G19 anti-TLA or mAb D10 anti-hair cell antigen (Richardson et al., 1990) hybridoma supernatants. mAb D10 is an $\mathrm{mAb}$ of the same subclass $\left(\mathrm{IgG}_{1}\right)$ as $\mathrm{G} 19$ that specifically recognizes sensory hair bundles in the inner ear and does not stain retina. The beads were collected by sedimentation at $1 \times g$ and washed six times with PBS/ $0.1 \%$ Triton $\mathrm{X}-100$, and bound proteins were eluted by heating to $100^{\circ} \mathrm{C}$ for $4 \mathrm{~min}$ in SDS-PAGE sample buffer. Eluted proteins were resolved on $6 \%$ SDS polyacrylamide gels and transferred to $\mathrm{Hy}-$ bondP using semidry blotting. The blots were preblocked in TBS containing $0.05 \%$ Tween- 20 and 3\% low-fat milk powder, incubated overnight in preblocking solution containing polyclonal antibodies PB473-3, PB303, or HL5383 at $0.5 \mu \mathrm{g} / \mathrm{ml}$ or polyclonal mouse anti-chick protocadherin-15-CD1 intracellular domain serum (M110) at a dilution of 1:2000, washed and reacted with alkaline phosphataseconjugated goat anti-rabbit or goat anti-mouse Ig (both at 1:1000 dilution; Dako, High Wycombe, UK) for $2 \mathrm{~h}$. Bound antibodies were visualized with nitroblue-tetrazolium-chloride/5-bromo-4-chlor-indolyl-phosphate (Roche Diagnostics, Lewes, UK).

Immunogold electron microscopy. Utricular maculae were rapidly dissected from the inner ear of P2 CD1 mice in HEPES-buffered ( $10 \mathrm{~mm}$ $\mathrm{pH}$ 7.2) HBSS (HBHBSS) and fixed for $1 \mathrm{~h}$ in $4 \%$ paraformaldehyde in $0.1 \mathrm{~m}$ sodium phosphate, $\mathrm{pH}$ 7.4. For pre-embedding labeling with PB375, the samples were washed in PBS, preblocked and permeabilized in TBS containing $0.1 \%$ Triton $\mathrm{X}-100$ for $1 \mathrm{~h}$, and incubated overnight at $4^{\circ} \mathrm{C}$ in the same solution containing affinity-purified PB375 or non-immune rabbit IgG at a concentration of $10 \mu \mathrm{g} / \mathrm{ml}$. After extensive washing, the samples were incubated overnight in goat anti-rabbit IgG conjugated to $5 \mathrm{~nm}$ colloidal gold (British Biocell International, Cardiff, UK) at a dilution of 1:10, washed, refixed in $2.5 \%$ glutaraldehyde in $0.1 \mathrm{M}$ sodium cacodylate containing $0.5 \%$ ruthenium red, washed in buffer, and postfixed in $1 \% \mathrm{OsO}_{4}$. Samples were dehydrated in ethanol, embedded in Epon, and sectioned at a thickness of 100 or $200 \mathrm{~nm}$. Chicken utricular maculae were double labeled with mAb G19 and rabbit anti-PB473-3 using a similar protocol except that a mixture of goat anti-mouse and goat anti-rabbit IgGs conjugated, respectively, to 10 and $5 \mathrm{~nm}$ colloidal gold were used to detect the primary antibodies. Controls with just one primary antibody (G19 or PB473-3) and both gold conjugates were used to validate the specificity of labeling.

For postembedding labeling, paraformaldehyde-fixed mouse maculae were dehydrated with cold ethanol and infiltrated with cold Unicryl resin overnight. The maculae were placed in BEEM capsule caps with fresh Unicryl resin, the resin-filled caps were covered with a plastic coverslip, and the resin was polymerized in the cold by exposure to UV light. Thin sections were cut and stained with PB375, PB303, or non-immune rabbit IgG at a concentration of $10 \mu \mathrm{g} / \mathrm{ml}$ followed by goat anti-rabbit IgG conjugated to $10 \mathrm{~nm}$ colloidal gold as described previously (Thorpe, 1999). Sections were counterstained with uranyl acetate and lead citrate and viewed in a Hitachi (Wokingham, UK) 7100 microscope operating at 75 or $100 \mathrm{kV}$, and images were captured with a Gatan (Abington, UK) Ultrascan 1000 CCD camera. For pre-embedding labeling, a minimum of four tissue blocks were sampled at several levels for each condition, and, for postembedding labeling, a minimum of 20 sections were examined with each antibody.

Treatment of cochlear cultures with BAPTA and subtilisin. Cochlear 
cultures were prepared from the inner ears of P1-P2 mouse pups on collagen-coated round glass coverslips as described previously (Russell and Richardson, 1987) and grown overnight in Maximow slide assemblies in a medium containing 93\% DMEM/F-12, 7\% fetal calf serum, and $10 \mu \mathrm{g} / \mathrm{ml}$ ampicillin. Cultures were removed from the Maximow slides, placed in 35mm-diameter plastic Petri dishes, and washed once with $3 \mathrm{ml}$ of HBHBSS (for subsequent saline or subtilisin treatment) or $3 \mathrm{ml}$ of $\mathrm{Ca}^{2+}$ free HBHBSS (for subsequent BAPTA treatment). Cultures were then treated with $\mathrm{HBH}-$ BSS, HBHBSS containing $50 \mu \mathrm{g} / \mathrm{ml}$ subtilisin (Protease XIV; Sigma, Poole, UK), or $\mathrm{Ca}^{2+}$ free HBHBSS containing $5 \mathrm{~mm}$ BAPTA for $15 \mathrm{~min}$ at room temperature before fixation in $4 \%$ paraformaldehyde in $0.1 \mathrm{~m}$ sodium phosphate buffer, pH 7.4. To examine the recovery of protocadherin- 15 epitopes, the BAPTA treatment time was reduced to $5 \mathrm{~min}$, and cultures were washed three times over a 5 min period in a large volume of HBHBSS and then fixed or replaced in Maximow slides with medium and cultured for an additional 1,4 , or $24 \mathrm{~h}$ at $37^{\circ} \mathrm{C}$.

\section{Results}

Novel protocadherin-15 isoforms are expressed in the inner ear

Regions of conserved DNA sequence with open reading frames are located downstream from the exons encoding the previously reported (Ahmed et al., 2001) C-terminal cytoplasmic domain (CD1) of protocadherin-15 in the human, mouse, rat, chicken, and zebrafish. RT-PCR analyses reveal transcripts in the mouse inner ear and the human retina that have a subset of additional alternatively spliced exons encoding two novel cytoplasmic domains (CD2 and CD3). These alternatively spliced $P c d h 15$ transcripts use a subset of the exons encoding the signal sequence, the extracellular domain (up to 11 extracellular cadherin repeats), a single-pass transmembrane domain, and sequence encoding cytoplasmic domain $\mathrm{CD} 1, \mathrm{CD} 2$, or CD3 (Fig. 1a-c).

Each of the three cytoplasmic domain classes has a unique amino acid sequence (542, 379, and 319 residues, respectively). $\mathrm{CD} 1, \mathrm{CD} 2$, and $\mathrm{CD} 3$ also have unique $\mathrm{C}$ termini (STSL, NTAL, and MTKL, respectively) (Fig. 1) (supplemental Fig. S1a-c, available at www. jneurosci.org as supplemental material), which are type $1 \mathrm{PDZ}$ (postsynaptic density 95/Discs large/zona occludens-1)-binding consensus sequences (X-T-X-L) (Sheng and Sala, 2001). Among 10 species for which the different cytoplasmic domain cassettes (CD1, $\mathrm{CD} 2$, and CD3) of protocadherin-15 were compared, the amino acid sequence of CD3 is the most highly conserved (data not shown). Surprisingly, the three cytoplasmic domains of protocadherin-15 (CD1, CD2, and CD3) (Fig. 1) do not comprise an exhaustive list. In humans and mice, we also discovered the identical composites of alternatively spliced portions of exons encoding either CD1 and CD3, or CD1, CD2, and CD3 (supplemental Fig. S1, available at stainings. Scale bars, $5 \mu \mathrm{m}$.
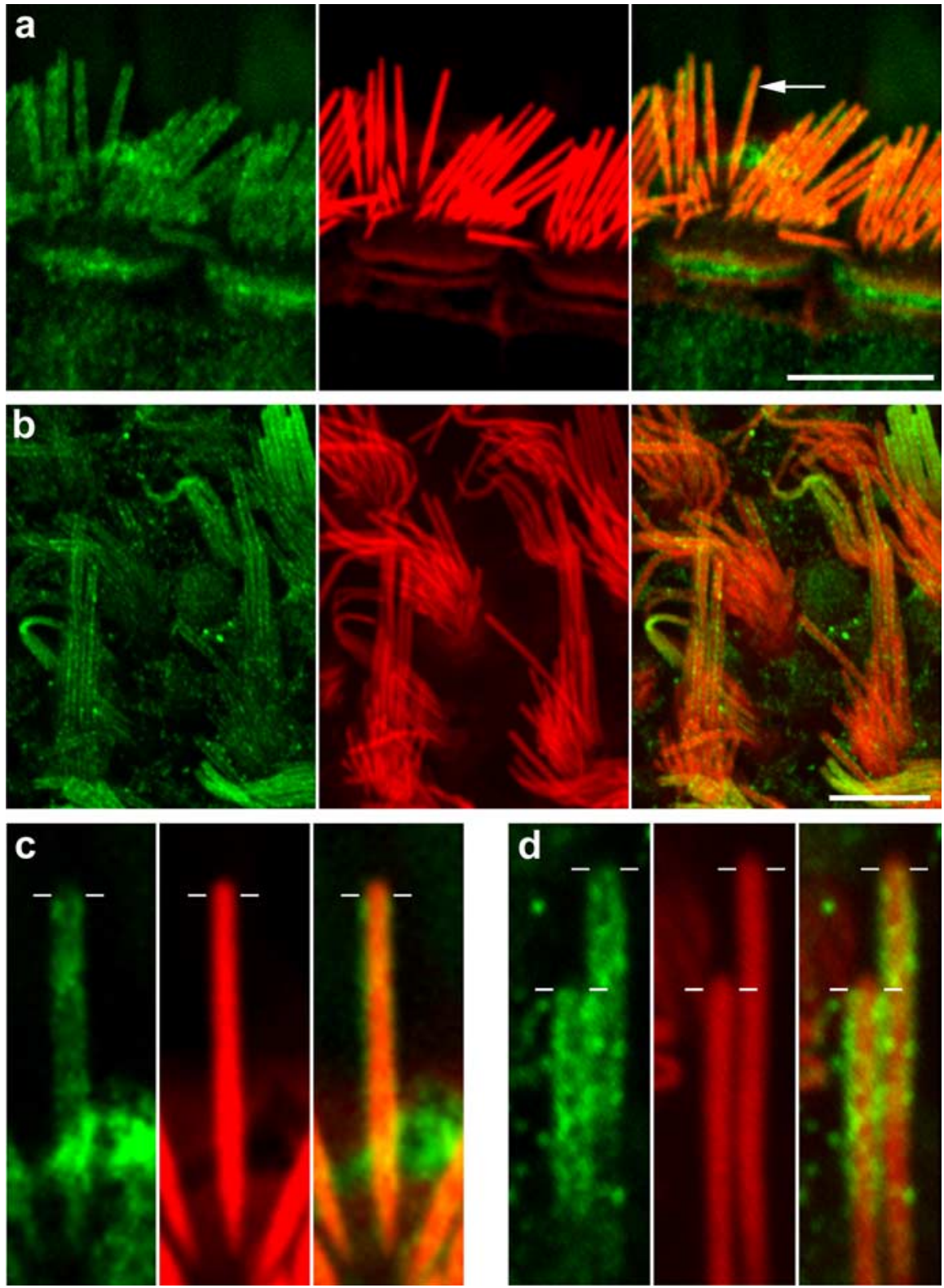

Figure 3. Distribution of protocadherin-15-CD1 in the hair bundle. $\boldsymbol{a}-\boldsymbol{d}$, Confocal images of mouse hair bundles double labeled with antibody PB303 directed against protocadherin-15-CD1 (green) and phalloidin to detect F-actin (red). $\boldsymbol{a}$, Adult mouse cochlea, inner hair cells. Arrow indicates the stereocilium shown enlarged in $\boldsymbol{c}$. $\boldsymbol{b}$, Adult mouse utricle. $\boldsymbol{c}$, Detail of adult mouse inner hair cell stereocilium. $\boldsymbol{d}$, Adult mouse utricle, detail of stereocilia. In $\mathbf{c}$ and $\boldsymbol{d}$, note that the tips of the stereocilia are devoid of staining for CD1 (absence of green fluorescence above the dashes). In $\boldsymbol{a}-\boldsymbol{d}$, the left panel shows the distribution of protocadherin-15-CD1, the middle panel shows the distribution of F-actin, and the right panel shows the merge of the two

www.jneurosci.org as supplemental material). In addition, a class of transcripts of Pcdh15 (protocadherin-15-SId) is expressed in the mouse inner ear that lacks the exons encoding the transmembrane and cytoplasmic domains and is likely to produce secreted isoforms of protocadherin-15 (Fig. 1d) (Rouget-Quermalet et al., 2006).

\section{Protocadherin-15 isoforms have different spatiotemporal expression patterns}

The distribution of the different isoform classes in the hair bundles of the developing inner ears of rats and mice was examined using antibodies generated against peptide sequences unique to each isoform class. The specificity of each antiserum for protocadherin-15 was verified by an absence of staining in the 

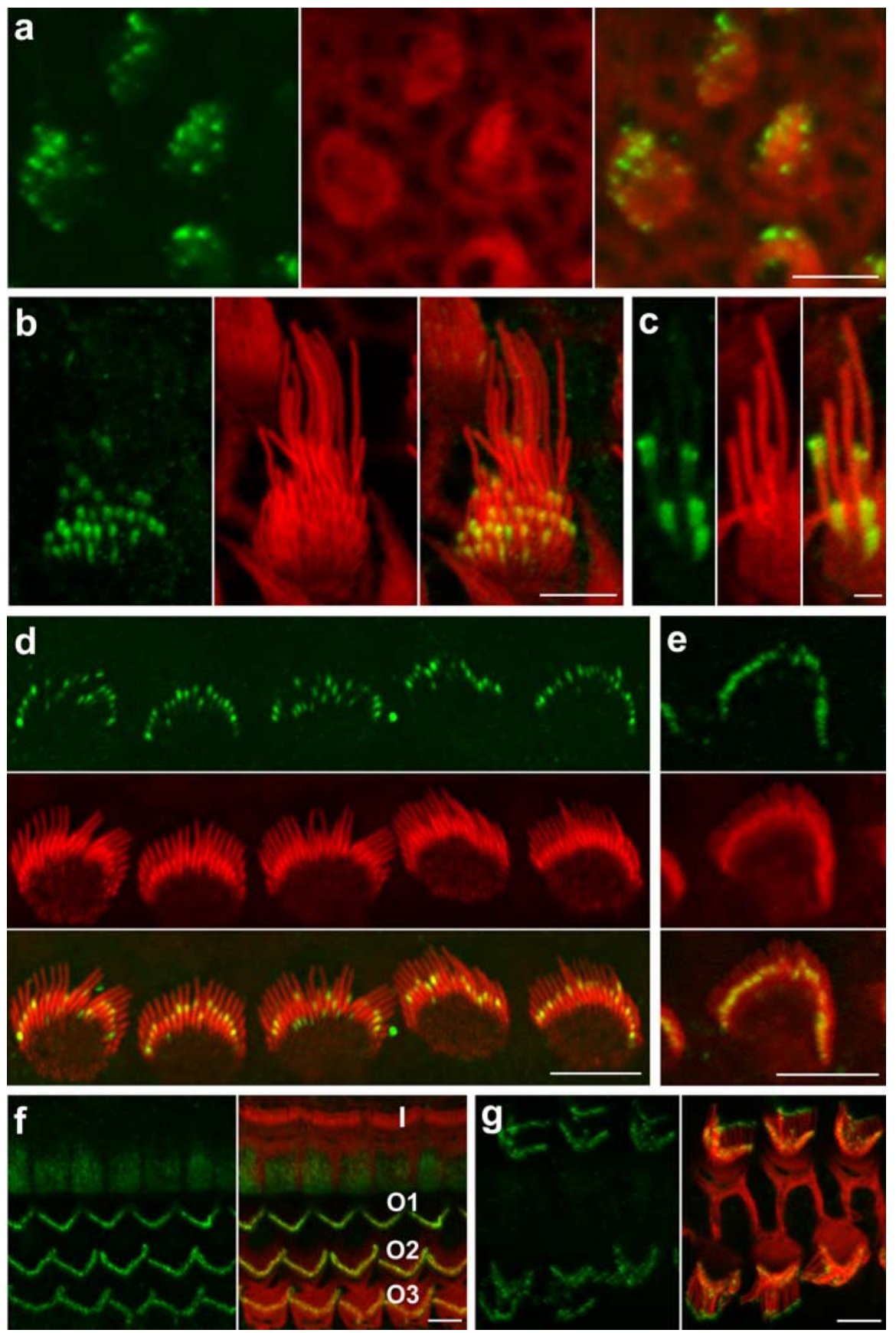

Figure 4. Protocadherin-15-CD3 distribution in hair bundles. $\boldsymbol{a}-\boldsymbol{g}$, Confocal images of hair bundles double labeled with antibodies to protocadherin-15-CD3 (PB375 or HL5383; green) and phalloidin (red). In $\boldsymbol{a}-\boldsymbol{e}$, the left or top panel shows the distribution of protocadherin-15-CD3, the middle panel shows the distribution of F-actin, and the right or bottom panel shows the merge of the two labels. In $\boldsymbol{f}$ and $\boldsymbol{g}$, the left panels shows protocadherin-15-CD3, and the right panel shows the merge with F-actin. $\boldsymbol{a}$, E15 mouse ampulla, antibody PB375. $\boldsymbol{b}$, P9 rat saccule, detail of the hair bundle, antibody PB375. c, P8 mouse utricle, detail of stereocilia tips, antibody PB375. $\boldsymbol{d}$, Inner hair cell stereocilia bundles, mouse organ of Corti, P2 plus $1 \mathrm{~d}$ in vitro, antibody PB375. e, Outer hair cell stereocilia bundle, mouse organ of Corti, P2 plus $1 \mathrm{~d}$ in vitro, antibody PB375.f, P17 rat organ of Corti, basal turn, antibody HL5383. Note that the stereocilia of the single row of inner hair cells (top of the image, I) lack staining, whereas the $V$-shaped stereocilia of the three rows of outer hair cells $(01,02,03)$ have protocadherin-15-CD3 immunoreactivity in the hair bundles. $g$, P17 rat organ of Corti, apical turn, antibody HL5383. Note that the tips of the stereocilia in all rows within the bundle are stained. Scale bars: $\boldsymbol{a}, \boldsymbol{b}, \boldsymbol{d}-\boldsymbol{g}, 5 \mu \mathrm{m} ; \boldsymbol{c}, 1 \mu \mathrm{m}$.

hair bundles of homozygous av-3J mice (supplemental Fig. S2, available at www.jneurosci.org as supplemental material). In cell lines transfected with either an epitope-tagged expression construct of protocadherin-15-CD1 (the last five ECs were deleted) or epitope-tagged forms of the protocadherin-15 cytoplasmic domains CD1, CD2, and CD3, the staining pattern observed with each antiserum was abolished by preincubating each serum with the peptide to which it was raised but not by a mixture of the peptides specific for the other two isoforms (supplemental Fig. S3a-f, available at www. jneurosci.org as supplemental material).

In the cochlea, hair cells in the basal, high-frequency end of the duct differentiate before those in the apical, low-frequency region, and, at any one stage of embryonic or early postnatal development, a gradient of hair-bundle maturation is seen along the length of the cochlea. In cochlear cultures at the equivalent of postnatal day 3 , clearly opposing gradients of staining are observed with antibodies specific for CD1 (Fig. $2 a-c$ ) and CD2 (Fig. 2d-f). Antibodies to CD1 do not stain hair bundles at the apical end of the apical coil (Fig. 2a), stain those in the middle of the apical coil (Fig. 2b), and react intensely with those in the basal coils (Fig. 2c). Antibodies to CD2 stain hair bundles in the apex of the cochlea intensely at this stage of development (Fig. $2 d$ ), those in the middle of the apical coil stain weakly (Fig. $2 e$ ), and, at the base of the cochlea, staining is barely detectable (Fig. 2f). With antibodies specific for CD3, a gradient of hair-bundle staining is observed that is similar to that seen with antibodies to CD1, i.e., little or no staining is seen at the apex (Fig. $2 g$ ), moderate staining is seen in the middle (Fig. $2 h$ ), and strong staining is seen in the base (Fig. 2i).

In mature hair bundles, protocadherin-15-CD1 is distributed fairly evenly along most of the length of the stereocilia on auditory hair cells (Fig. 3a), whereas it is concentrated toward the upper third of the hair bundle in vestibular hair cells (Fig. $3 b$ ). In both the auditory and the vestibular organs, protocadherin-15-CD1 is excluded from a region at the very tip of each stereocilium (Fig. $3 c, d$ ). In contrast, protocadherin-15-CD2 immunoreactivity decreases to an undetectable level in adult mouse cochlear hair cells (data not shown). Immature and mature hair cells are both present in the sensory epithelium of the early postnatal rodent vestibule. The entire hair bundle of the immature cells stains uniformly and intensely for protocadherin-15-CD2, whereas only the kinocilium is stained in the more mature hair bundles (supplemental Fig. S4, available at www.jneurosci.org as supplemental material).

With antibody PB375 directed against a unique peptide sequence in protocadherin-15-CD3, staining is detected in immature vestibular hair bundles as early as embryonic day 15.5 (E15.5) and is concentrated toward the tip of each stereocilium (Fig. 4a). Protocadherin-15-CD3 also localizes to the tips of the 
shorter stereocilia in the mature vestibular hair bundles of adult mice and rats and is not detected at the tips of the stereocilia in the tallest row (Fig. $4 b, c$ ). In the more mature hair bundles at the base of the cultured mouse cochlea at the equivalent of P3, staining with PB375 is restricted to the tips of the shorter stereocilia in both inner (Fig. 4d) and outer (Fig. 4e) hair cells. A similar staining pattern is observed in hair bundles throughout most of the length of the cochlea at this stage but not in those of outer hair cells at the very apical end of the sensory epithelium (Fig. 2g). Protocadherin-15-CD3 immunoreactivity (PB375) is not detectable in the hair bundles of the mature cochlea using these antibodies (data not shown). An affinity-purified antibody (HL5383) directed against a GST-CD3 fusion protein (validated as described above) (supplemental Figs. S2, S3, available at www.jneurosci.org as supplemental material), however, detects protocadherin15-CD3 at the tips of all the stereocilia (shortest, intermediate, and longest) in outer hair cells from P10 onward, although not in inner hair cells (Fig. 4f,g). As yet, it is unclear why there are these differences in reactivity to various CD3 antisera, but they could be attributable to cell- and timedependent variations in posttranslational modifications or epitope accessibility.

The distributions of protocadherin-15CD1 and protocadherin-15-CD3 in vestibular hair cells were also examined with immunogold electron microscopy using anti-peptide antibodies PB303 and PB375 (Fig. 5). Consistent with the immunofluorescence staining, postembedding immunogold labeling reveals that protocadherin-15CD1 is essentially excluded from the tip region of hair-cell stereocilia (Fig. 5a,b). Very few gold particles are observed in sections stained with non-immune rabbit IgG (Fig. 5c). Both postembedding (Fig. 5d) and pre-embedding (Fig. 5e,f) immunogold labeling reveal that protocadherin-15-CD3 is distributed in a fairly uniform manner around the distal tips of the shorter stereocilia.

Two antibodies raised to sequences in the ectodomain of protocadherin-15 (validated as described above) (supplemental Figs. S2, S3, available at www.jneurosci.org as supplemental material) stain developing but not mature cochlear hair bundles (Fig. 6). One of these antibodies, PB473-3, an antibody to a peptide sequence located immediately upstream of the first cadherin repeat of protocadherin-15 (Fig. 1), only stains the stereocilia of developing mouse cochlear hair bundles in the presence of TX-100 (Fig. $6 a-c$ ). The other antibody, HL5614, raised to a recombinant fusion protein encompassing the first two cadherin repeats of protocadherin-15 (Fig. 1), only stains the stereocilia of developing mouse cochlear hair bundles if they are treated with the calcium

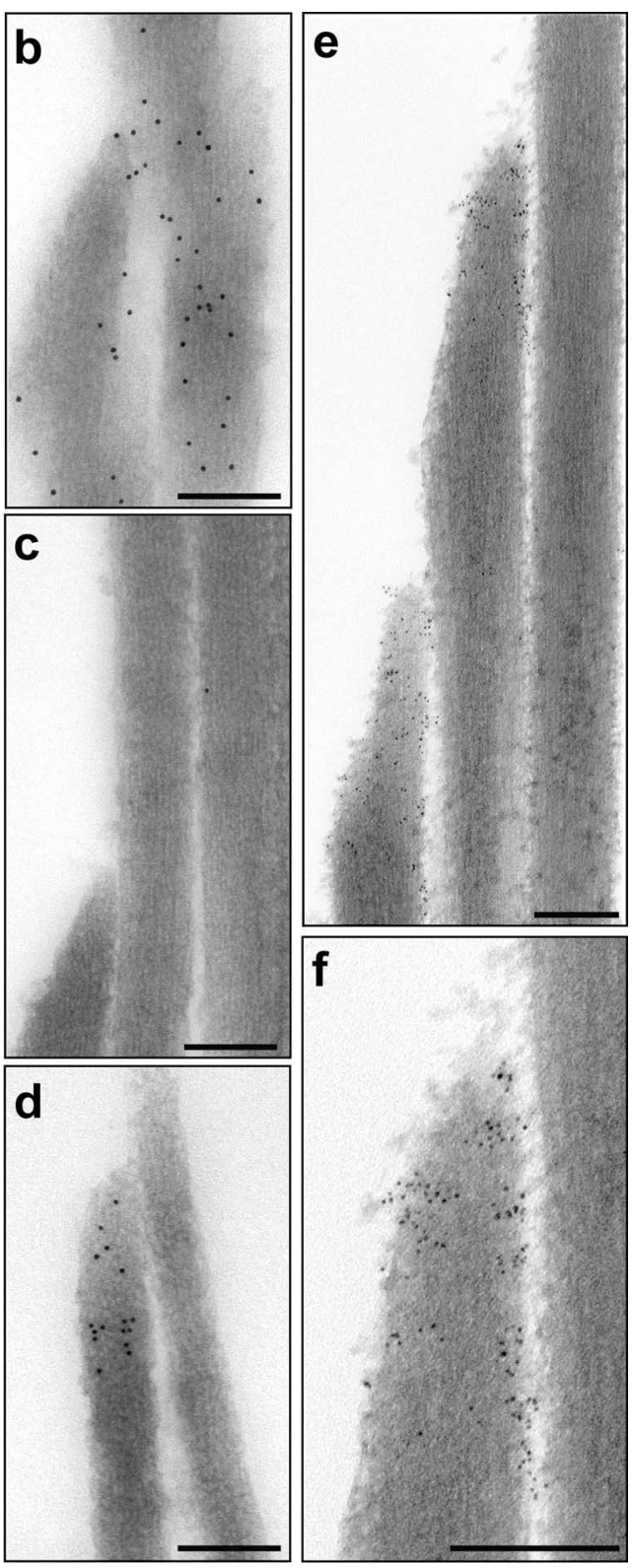

Figure 5. Immunogold electron microscopy of protocadherin-15-CD1 and protocadherin-15-CD3. Electron micrographs of P2 mouse utricle hair bundles stained with antibody PB303 to protocadherin-15-CD1 $(\boldsymbol{a}, \boldsymbol{b})$, non-immune rabbit lgG $(\boldsymbol{c})$, and antibody B375 to protocadherin-15-CD3 (d-f). Hair bundles were labeled using postembedding labeling with primary antibody followed conjugated goat anti-rabbit $\lg (\boldsymbol{e}, \boldsymbol{f}) . \boldsymbol{f}$ is an enlargement of the tip of the taller of the two stereocilia shown in $\boldsymbol{e}$. Note how protocadherin-15-CD1 is excluded from the very tip of the stereocilium $(\boldsymbol{a}, \boldsymbol{b})$ and how protocadherin-15-CD3 $(\boldsymbol{d}-\boldsymbol{f})$ is distributed around the tip of each stereocilium. Scale bars, $200 \mathrm{~nm}$.

chelator BAPTA before fixation (Fig. $6 d-f$ ). In the absence of BAPTA treatment before fixation, staining of the kinocilium is readily detectable. A pronounced apical-to-basal gradient of staining is observed in the early postnatal mouse cochlea with both ectodomain antibodies, with hair bundles in the apical coil (Fig. 6b,e) staining more intensely than those in the basal coil (Fig. $6 c, f$ ), as observed with antibodies specific for protocadherin-15-CD2 (Fig. 2d-f).

\section{The avian tip-link antigen is protocadherin-15}

The TLA mAb G19, a species-specific antibody that recognizes an extracellular epitope specifically associated with the tip and kino- 

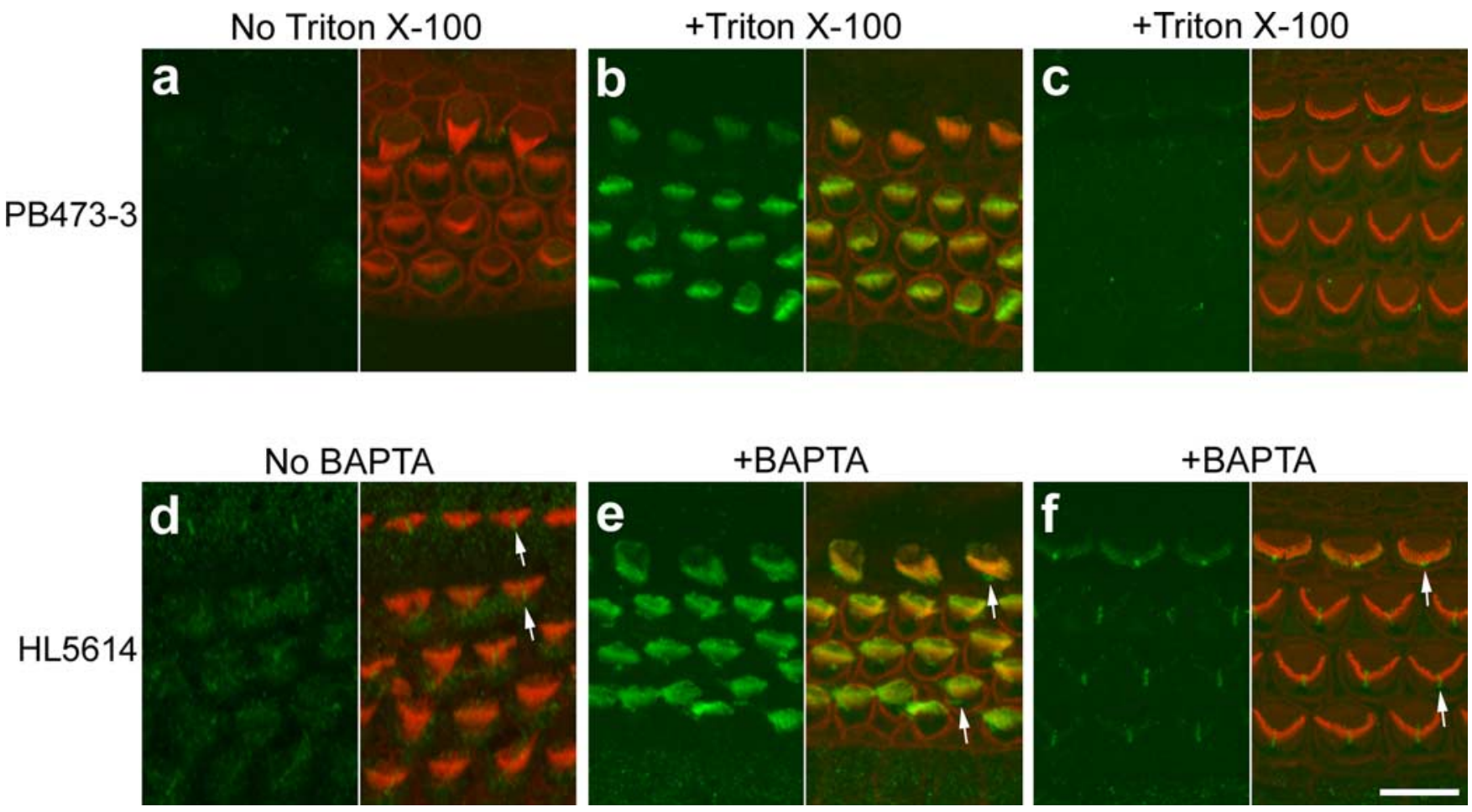

Figure 6. Distribution of protocadherin-15 ectodomain epitopes in cochlear hair bundles. $\boldsymbol{a}-\boldsymbol{f}, \mathrm{P2}$ mouse organ of Corti, $1 \mathrm{~d}$ in vitro, double labeled with antibody PB473-3 (a-c, green) or antibody HL5614 (d-f, green) and phalloidin (red). The left half of each panel shows the distribution of protocadherin-15, and the right half shows the merge with F-actin. $\boldsymbol{a}$, Apical coil, antibody PB473-3, without TX-100.b, Apical coil, antibody PB473-3, with TX-100.c, Basal coil, antibody PB473-3, with TX-100.d, Apical coil, antibody HL5614, without BAPTA.e, Apical coil, antibody HL5614, after BAPTA. $f$, Basal coil, antibody HL5614, after BAPTA. Kinocilia are indicated by arrows. Scale bar, $10 \mu \mathrm{m}$.

\section{Table 1. Mass spectrometric sequencing of chicken TLA}

\begin{tabular}{|c|c|c|c|c|}
\hline Number & Peptide sequence & Protocadherin-15 location & TLA1 & TLA2 \\
\hline 1 & TGPPATIVPIDEESR & $\mathrm{N}$ terminus & + & + \\
\hline 2 & GTAAGPDPTIELSLK & $\mathrm{EC}_{1}$ & + & - \\
\hline 3 & DNVDYWVILDPISQR & $\mathrm{EC}_{1}$ & - & + \\
\hline 4 & YFVIVQANDR & $\mathrm{EC}_{2}$ & + & + \\
\hline 5 & YLTLLQPVDR & $\mathrm{EC}_{4}$ & + & + \\
\hline 6 & TGAILLNLQAFDR & $\mathrm{EC}_{6}$ & + & + \\
\hline 7 & TQEALEYFALNK & $\mathrm{EC}_{8}$ & - & + \\
\hline 8 & YTGELSLLK & $\mathrm{EC}_{8}$ & - & + \\
\hline 9 & DMNDYSPVFSK & $\mathrm{EC}_{8}$ & - & + \\
\hline 10 & VNLNEEPSTVFK & $\mathrm{EC}_{9}$ & - & + \\
\hline 11 & LVVIAYDDGDPVK & $\mathrm{EC}_{9}$ & + & - \\
\hline 12 & FQVIATDDYGK & $\mathrm{EC}_{11}$ & + & + \\
\hline 13 & DQLIGILER & between $\mathrm{EC}_{11}$ and TM & - & + \\
\hline 14 & YVQDQIPGATVVVESIGAR & between $\mathrm{EC}_{11}$ and TM & - & + \\
\hline 15 & VDISSPLFQK & CD1 & + & - \\
\hline
\end{tabular}

Peptide sequences identified in the chicken genome from tryptic digests of the TLA1 and TLA2 bands. The peptides highlighted in bold are conserved in huma and mouse protocadherin-15. TM, Transmembrane domain.

cilial links of chick hair cells and the calyceal processes of photoreceptors, immunoprecipitates two proteins of $\sim 200$ and 250 $\mathrm{kDa}$ from chicken retina (Goodyear and Richardson, 2003). These relative mobilities are similar to those predicted for the longer isoforms of protocadherin-15. To identify these TLA immunoreactive proteins, they were immunopurified from chicken retina, resolved by one-dimensional gel electrophoresis, and analyzed by mass spectrometry (LC-MS/MS). Two peptide sequences from each of the bands (TLA1 and TLA2) match sequences corresponding to two evolutionarily conserved regions of the extracellular domain of human, mouse, and chicken protocadherin-15 [YLTLLQPVDR (GenBank accession numbers NP_149045, AAG53891; MASCOT score of 75) and FQVIATD-
DYGK (GenBank accession numbers DQ354419; MASCOT score of 85)]. Using the same LC-MS/MS data files, a search of the chicken proteome identifies 8 and 12 peptides, respectively, from the 250 and $200 \mathrm{kDa}$ TLA proteins (Table 1; Fig. 7b, red rectangles). All of these are statistically significant matches to chicken protocadherin-15 and are unique peptides in the chicken proteome. One of these peptides was located in the CD1 domain of chicken protocadherin-15.

Data from Western blot analyses confirm these findings, revealing that the two bands immunoprecipitated by mAb G19 from the avian retina (Fig. $7 a$, bands 1,2 ) are recognized by the antibody directed against the peptide in the extracellular domain of mouse protocadherin-15 (Fig. $7 a$, PB473-3). Mouse protocadherin-15 and the TLA are thus indistinguishable by Western blot analyses. The TLA1 band with the slower mobility is also recognized by the antibodies directed against the cytoplasmic domain of mouse protocadherin-15-CD1 (Fig. 7a, PB303) and by a polyclonal mouse antiserum directed against a recombinant fragment of chicken CD1 (Fig. 7a, M110), as expected from the mass spectrometric analysis (Table 1). Antibodies directed against mouse protocadherin-15-CD3 detect a minor band that is also immunoprecipitated by the TLA mAb and migrates just slightly faster than the upper TLA1 band (Fig. 7a, HL5383). The TLA2 band is not recognized by antibodies to mouse or chick CD1 (Fig. 7a, PB303, M110) or an antibody to mouse protocadherin-15-CD3 
(Fig. 7a, HL5383), although it does react with the antibody to the peptide in the ectodomain of mouse protocadherin-15 (Fig. 7a, PB473-3). The TLA2 band also does not react with the antibody (PB464$2 \mathrm{~B})$ to mouse protocadherin-15-CD2 (data not shown). As in mouse, and as expected from Western blotting, multiple transcripts of protocadherin-15 are expressed in the chick. Using poly $(\mathrm{A})^{+}$RNA from chicken brain, transcripts of chicken protocadherin-15 can be detected that encode CD1, a fusion of the cytoplasmic domain sequence of CD2 and CD3 (CD2/3), and a CD3 cytoplasmic domain (Fig. $7 b-d)$.

The PB473-3 antibody directed against the extracellular peptide epitope in mouse protocadherin-15 was used in doublelabeling experiments of chicken utricle with mAb G19. Confocal microscopy reveals that both antibodies immunolocalize protocadherin- 15 to the distal region of the kinocilium and bright spots near the tips of the stereocilia (Fig. $8 a-c$ ). The staining pattern observed with the two antibodies is very similar, although mAb G19 stains the kinocilium with a greater intensity than antibody PB473-3. Immuno-EM using two different sizes of gold particles indicates that both antibodies stain the tip- and kinocilial-link regions of the hair bundle (Fig. $8 d-f$ ).

\section{Protocadherin-15-CD3 reappears after BAPTA treatment}

The four link types that interconnect the stereocilia can be distinguished by their relative sensitivities to proteolysis with subtilisin and calcium chelation with BAPTA. Tip links are BAPTA-sensitive/ subtilisin-resistant structures, horizontal top connectors are unaffected by treatment with either agent, shaft connectors are subtilisin-sensitive/BAPTA-resistant structures, and ankle links are abolished by treatment with either BAPTA or subtilisin (Goodyear and Richardson, 1999; Goodyear et al., 2005). Kinocilial links are, like tip links, BAPTA-sensitive/ subtilisin-resistant structures (Goodyear and Richardson, 2003). Tip links reappear after the removal of BAPTA with a recovery time constant of $\sim 4 \mathrm{~h}$, and mechanotransduction is restored over a similar time course (Zhao et al., 1996).

Mouse cochlear cultures were therefore used to examine how calcium chelation and exposure to the protease subtilisin affect the distribution of the different protocadherin-15 isoforms in the hair bundle (Fig. 9). The distribution of protocadherin-15-CD1 (Fig. $9 a-c$ ) and protocadherin-15-CD2 (Fig. 9d-f) are unaffected by treatment with either BAPTA or subtilisin, but both agents cause a loss of protocadherin-15-CD3 immunoreactivity from the stereocilia (Fig. $9 g-i$ ). The ectodomain epitopes detected by the anti-peptide antibody PB473-3 in the presence of TX-100 are unaffected by either calcium chelation or subtilisin treatment (data not shown), whereas those detected by antibody
HL5614 to the recombinant fusion protein that require calcium chelation for visualization are subtilisin sensitive (Fig. 9j-l).

The ectodomain epitopes that are revealed by calcium chelation rapidly disappear when calcium is replaced, within $5 \mathrm{~min}$ at room temperature after a 5 min exposure to BAPTA (Fig. 10a,b). This is most likely attributable to rapid remasking rather than shedding or loss of the ectodomain because a subsequent, second brief BAPTA treatment reveals the epitopes recognized by this antibody again (Fig. 10c). Protocadherin-15-CD3 reappears more slowly after BAPTA treatment. None is detectable after a $1 \mathrm{~h}$ recovery period at $37^{\circ} \mathrm{C}$, and complete recovery is observed within $4-24 \mathrm{~h}$ (Fig. $10 d-f$ ).

\section{Discussion}

The results reveal that multiple protocadherin-15 transcripts are expressed in the mouse inner ear and define four major isoform classes, two of which have entirely novel and previously unidentified cytoplasmic domains. Antibodies specific for the three alternative cytoplasmic domains reveal that three of the four iso- 

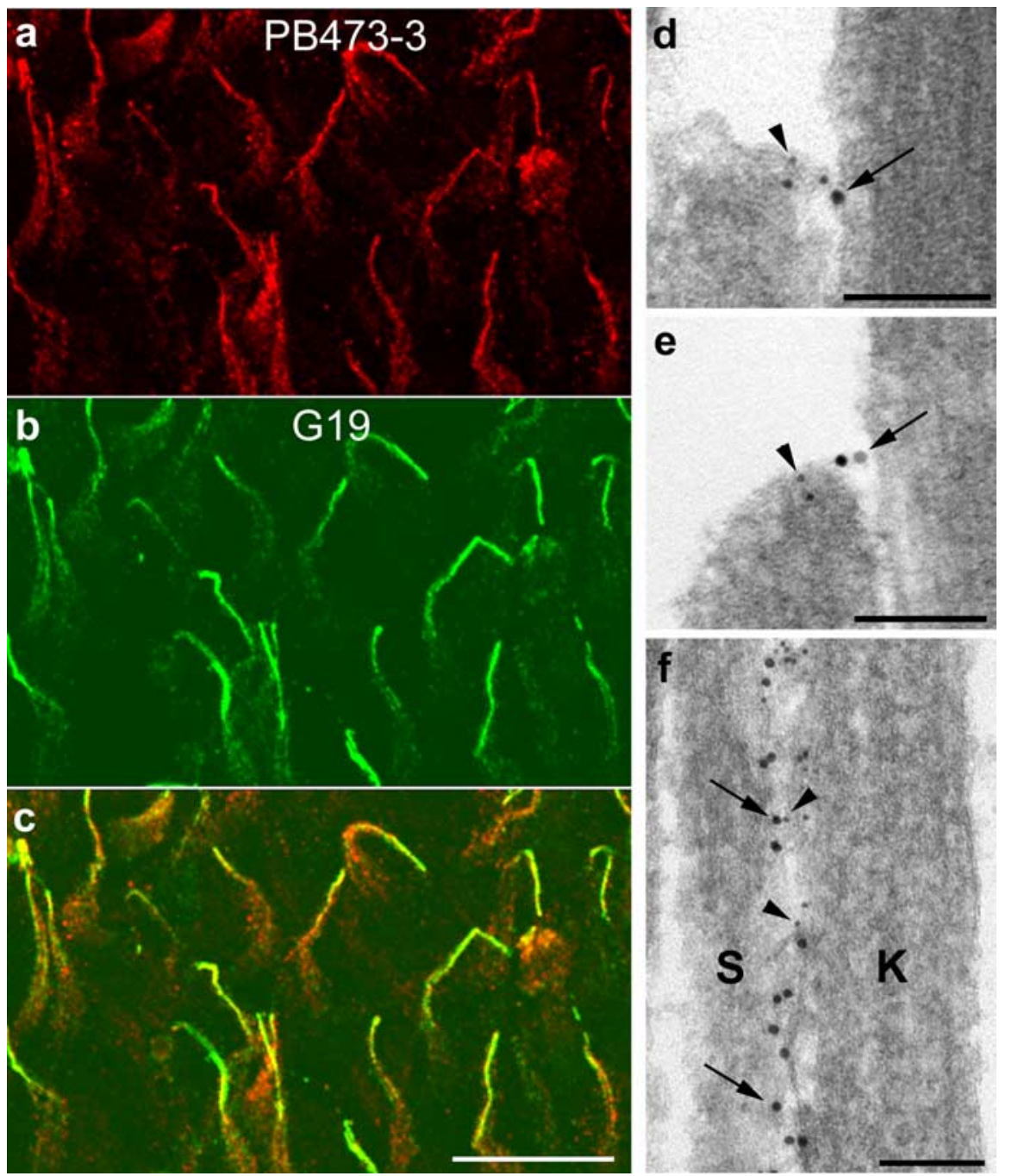

Figure 8. Colocalization of the TLA and protocadherin-15. $\boldsymbol{a}-\boldsymbol{c}$, Confocal images of the chicken utricular macula double labeled for the PB473-3 peptide ( $\boldsymbol{a}$, red) and the TLA using MAb G19 $(\boldsymbol{b}$, green). Image in $\boldsymbol{c}$ is a merge of images shown in $\boldsymbol{a}$ and $\boldsymbol{b}$. Scale bar, $10 \mu \mathrm{m} . \boldsymbol{d}-\boldsymbol{f}$, Double-immunogold labeling for the PB473-3 peptide (5 nm particles; arrowheads) and the TLA (10 nm particles; arrows) in the tip-link region $(\boldsymbol{d}, \boldsymbol{e})$ and in the stereocilia-kinocilial link region $(\boldsymbol{f})$ of utricular macular hair cells. S, Stereocilium; $\mathrm{K}$, kinocilium. Scale bars, $100 \mathrm{~nm}$.

form classes have very different spatiotemporal expression patterns in developing and mature hair cells (supplemental Fig. S5, available at www.jneurosci.org as supplemental material), indicating a specific role for each protocadherin-15 isoform class in the ontogeny, structure, and function of the sensory hair bundle.

Isoforms from one of the novel classes, protocadherin-15$\mathrm{CD} 2$, are expressed along the lengths of stereocilia during the early stages of hair-bundle development, but become progressively localized toward the tip of the bundle in more mature hair cells and are then lost from the stereocilia. Isoforms from this class are not expressed by mature cochlear hair cells and are only present in the kinocilium of mature vestibular hair cells. The gradient of staining that diminishes toward the basal turn of the cochlea in the early postnatal cochlea supports the possible involvement of protocadherin-15-CD2 in the formation of the transient lateral links that are found in abundance on developing cochlear hair bundles (Goodyear et al., 2005). This expression pattern is similar to that described previously for cadherin-23 (Lagziel et al., 2005; Michel et al., 2005) and suggests that cadherin-23 and protocadherin-15-CD2 may contribute collec- tively to the transient lateral links and also to the kinocilial links of mature vestibular hair cells.

The previously reported protocadherin-15-CD1 is not found on the surface of immature cochlear hair bundles at the apex of the early postnatal (P3) mouse cochlea and is therefore unlikely to play a role in the early stages of hair-bundle development. In mature hair cells, protocadherin-15-CD1 is not found on the kinocilium (when present) and, although concentrated toward the tops of the stereocilia in vestibular hair cells, is clearly excluded from the extreme tip region of each stereocilium in both auditory and vestibular hair cells. In contrast to a recent study (Senften et al., 2006), protocadherin-15CD1 was never found to be concentrated in the ankle-link region of the hair bundle, even during the postnatal stages of development (P2-P9) when ankle links are prominent (Goodyear et al., 2005).

An isoform from another novel class, protocadherin-15-CD3, is concentrated at the tips of developing stereocilia and expressed along the length of their shafts but becomes restricted to the tips of stereocilia as the hair bundle matures. The mechanisms that direct the various protocadherin-15 isoforms to different domains within the hair bundle remain to be elucidated but may involve molecules that interact with the unique PDZ ligands or other motifs exclusive to each of the cytoplasmic domains. Harmonin, a scaffold protein that has many alternative splice isoforms, and myosin VIIA have been shown to interact in vitro with the CD1 domain of protocadherin-15 (Adato et al., 2005; Reiners et al., 2005; Senften et al., 2006) and may be involved in the localization of protocadherin- 15 in stereocilia.

Protocadherin-15-CD1 and protocadherin-15-CD3 are both expressed in the mature hair bundle, but neither is distributed in a manner that indicates they are uniquely associated with one particular link type found in mature hair bundles. These isoforms are, however, found where the basal end of the tip link attaches to the tip of the shorter stereocilium (CD3) and where the distal end attaches to the side of the adjacent taller stereocilium (CD1), so both isoforms could potentially be associated with the tip-link complex (supplemental Fig. S5, available at www.jneurosci.org as supplemental material). The results of this study further show that the TLA, a previously unidentified antigen known to be specifically associated with the tip and kinocilial links of sensory hair bundles in the chick inner ear and the ciliary calyx of photoreceptors in the eye (Goodyear and Richardson, 2003), is an avian ortholog of human protocadherin-15, a product of the deaf/ blindness Usher syndrome type 1F/DFNB23 locus (Ahmed et al., 2001, 2003; Alagraman et al., 2001b). One of the two TLA bands is a mixture of two isoforms, one reactive with antibodies to mouse and chick protocadherin-15-CD1 and the other reactive with antibodies to mouse protocadherin-15-CD3. 
An antibody raised to the peptide sequence potentially common to the ectodomain of all mouse protocadherin-15 isoform classes stains chick hair bundles in a manner similar to that observed with the anti-TLA mAb, further confirming the TLA is protocadherin-15. Although this antibody and a second antiserum raised to a recombinant fragment encompassing the same peptide and the first two cadherin repeats (also expected to react with isoforms from all four classes of protocadherin-15) both reacted intensely with embryonic mouse hair bundles, they failed to stain mature mouse hair bundles. In the developing mouse cochlea, reactivity to these antibodies diminished rapidly during the early postnatal stages of development, and the spatiotemporal staining pattern observed with these antibodies is similar to that observed with the antibody raised to the cytoplasmic domain of protocadherin-15-CD2. The protocadherin$15-\mathrm{CD} 1$ and protocadherin-15-CD3 isoforms expressed in the mouse may lack the regions of sequence recognized by these ectodomain antibodies, or these regions may somehow be masked and inaccessible in the mouse. In this respect, it should be noted that TX-100 was, for reasons that are as yet unclear, required to visualize protocadherin- 15 with the antibody to the extracellular peptide sequence and that BAPTA treatment was necessary to detect protocadherin-15 with the antibody to the recombinant ectodomain fragment. In the mouse, BAPTA and TX-100 appear to only unmask the epitopes associated with the ectodomain of protocadherin-15CD2 and not those associated with the ectodomains of protocadherin-15-CD1 or protocadherin-15-CD3.

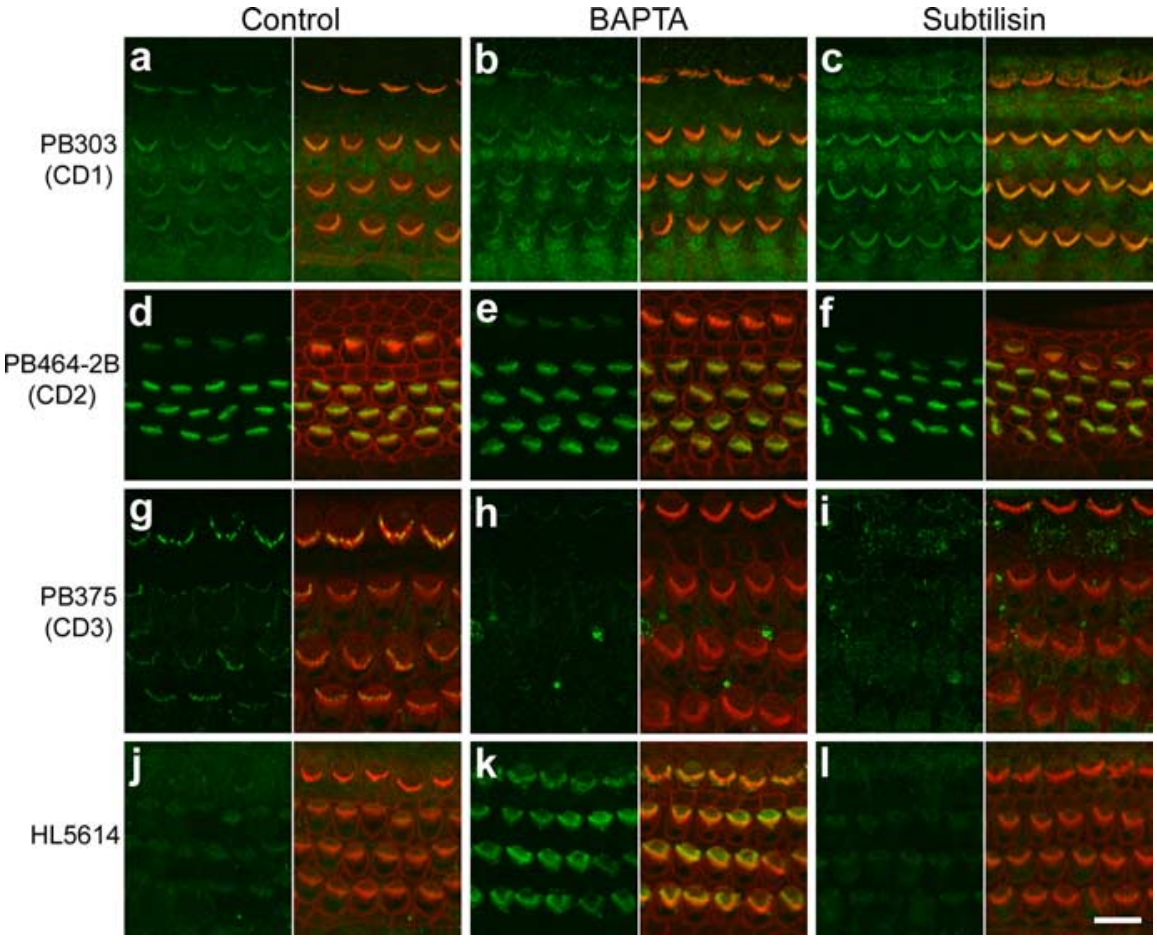

Figure 9. Effects of BAPTA and subtilisin on protocadherin-15 isoform classes. $a-I$, Mouse organ of Corti, P2 plus $1 \mathrm{~d}$ in vitro, double labeled with phalloidin for F-actin (red) and antibodies (green) PB303 to protocadherin-15-CD1 (a-c), PB464-2B to protocadherin-15-CD2 $(\boldsymbol{d}-\boldsymbol{f})$, PB375 to protocadherin-15-CD3 $(\boldsymbol{g}-\boldsymbol{i})$, and HL5614 to a recombinant ectodomain fragment of protocadherin-15 (j-I). Cultures were treated with saline $(\boldsymbol{a}, \boldsymbol{d}, \boldsymbol{g}, \boldsymbol{j}$; Control), $5 \mathrm{~mm}$ BAPTA $(\boldsymbol{b}, \boldsymbol{e}, \boldsymbol{h}, \boldsymbol{k} ;$ BAPTA), or $50 \mu \mathrm{g} / \mathrm{ml}$ subtilisin $(\boldsymbol{c}, \boldsymbol{f}, \boldsymbol{i}, \boldsymbol{l} ;$; Subtilisin) for $15 \mathrm{~min}$ at room temperature. Culture in $I$ was exposed to BAPTA after subtilisin treatment to reveal masked epitopes for HL5614. The left half of each panel shows the distribution of protocadherin-15, and the right half shows the merge with F-actin. Scale bar, $10 \mu \mathrm{m}$.

The data discussed above indicate that protocadherin-15CD1 and protocadherin-15-CD3 may both be associated with the tip-link complex, and several other lines of evidence indicate that protocadherin-15 is required for mechanotransduction. First, receptor potentials cannot be detected from the lateral line
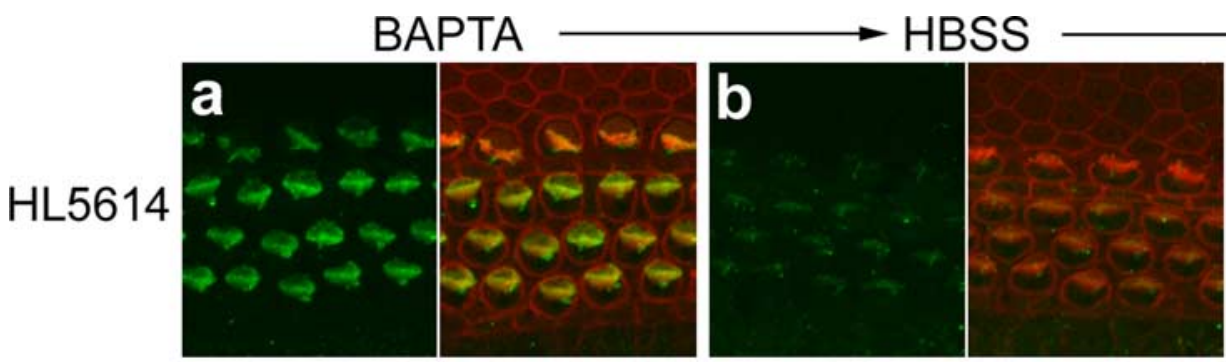

$1 \mathrm{hr}$ recovery
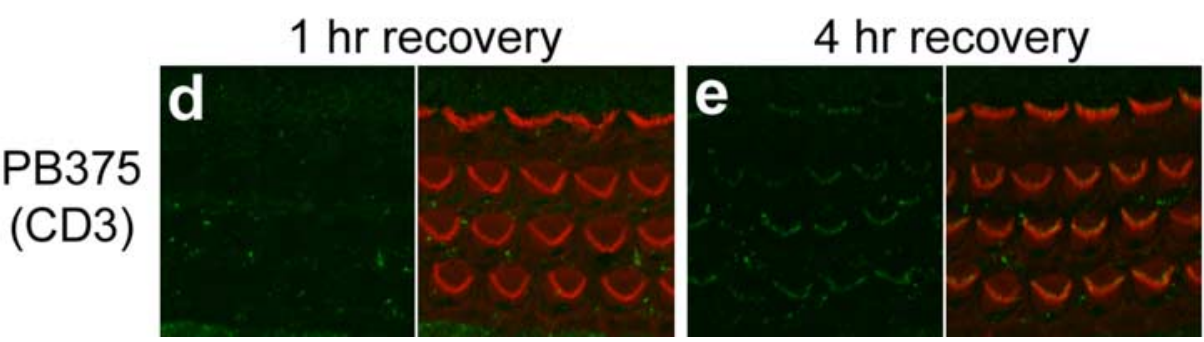
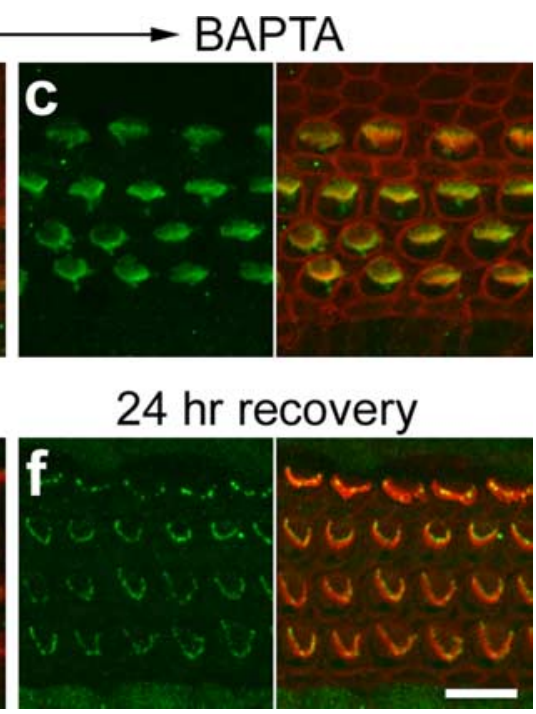

Figure 10. Reappearance of protocadherin-15 epitopes after BAPTA treatment. $\boldsymbol{a}-\boldsymbol{f}, \mathrm{P} 2$ mouse organ of Corti, $1 \mathrm{~d}$ in vitro, labeled with phalloidin for F-actin (red) and antibody HL5614 to a recombinant ectodomain fragment of protocadherin-15 ( $\boldsymbol{a}-\boldsymbol{c}$, green) and PB375 to protocadherin-15-CD3 ( $\boldsymbol{d}-\boldsymbol{f}$, green). Cultures in $\boldsymbol{a}-\boldsymbol{c}$ were treated as follows at room temperature: $\boldsymbol{a}, 5 \mathrm{mM}$ BAPTA for $5 \mathrm{~min} ; \boldsymbol{b}, 5 \mathrm{~mm}$ BAPTA for $5 \mathrm{~min}$, followed by saline for $5 \mathrm{~min} ; \boldsymbol{c}, 5 \mathrm{~mm}$ BAPTA for $5 \mathrm{~min}$, saline for $5 \mathrm{~min}$, followed by $5 \mathrm{~mm}$ BAPTA for $5 \mathrm{~min}$. Cultures in $\boldsymbol{d}-\boldsymbol{f}$ were treated with $5 \mathrm{~mm}$ BAPTA for $5 \mathrm{~min}$ at room temperature, washed in saline over a $5 \mathrm{~min}$ period, and then replaced in culture at $37^{\circ} \mathrm{C}$ for $1 \mathrm{~h}(\boldsymbol{d}), 4 \mathrm{~h}(\boldsymbol{e})$, and $24 \mathrm{~h}(\boldsymbol{f})$. The left half of each panel shows the distribution of protocadherin-15, and the right half shows the merge with F-actin. Scale bar, $10 \mu \mathrm{m}$. 
organs of zebrafish orbiter mutants with mutations in the extracellular domain of Pcdh15 despite normal-appearing hair bundles (Nicolson et al., 1998; Seiler et al., 2005). Second, vestibular evoked potentials cannot be detected in av mice with mutations in Pcdh15, and the mature utricular hair cells of the $a v-2 J$ and av-3J mutant do not load with AM1-43 (Alagramam et al., 2005), a fixable version of FM1-43 [ $N$-(3-triethylammoniumpropyl)-4(4-(dibutylamino)styryl) pyridinium dibromide], a styryl dye that is known to enter and accumulate in hair cells via their transducer channels (Gale at al., 2001; Meyers et al., 2003). Third, transduction currents cannot be recorded using whole-cell patch clamping in either the vestibular or cochlear hair cells of early postnatal $a v-3 J$ mice, and neither cell type loads with the fixable form of FM1-43, FM1-43FX (Senften at al., 2006). Fourth, one of the two isoform classes that is potentially associated with tip links, protocadherin-15-CD3, is rapidly lost from the hair bundle in response to calcium chelation, a condition that is known to abolish transduction (Assad et al., 1991). Protocadherin-15-CD3 also reappears over the period transduction (Zhao et al., 1996), and FM1-43 dye loading (Gale et al., 2001) recovers after the removal of calcium chelators. Although the results further indicate the ectodomain of protocadherin-15 can rapidly refold on the cell surface after removal of BAPTA, this is a very rapid process and one that foreshadows the recovery of transduction.

Despite this evidence, it remains debatable whether protocadherin- 15 is a component of the central strand of the tip link, a component of the "anchoring" elements of the tip link, peripherally associated with the tip-link complex, and/or required for tip-link formation. Tip links can be up to $250 \mathrm{~nm}$ in length and are composed of two helical filaments along most of their length (Kachar et al., 2000; Tsuprun et al., 2004). The predicted extracellular domain of protocadherin-15 has a juxtamembrane region with a mass of $53 \mathrm{kDa}$ and unknown tertiary structure and a distal region with a maximum of 11 EC repeats, each of which spans $4.3 \mathrm{~nm}$ (Boggon et al., 2002). Even as a trans dimer, protocadherin-15 is unlikely to be long enough to form a tip link. There is evidence that the tip link splays at both ends into two or more strands and that it is attached to the tip of the shorter stereocilium by three anchoring filaments (Kachar et al., 2000). In the mouse, protocadherin-15-CD3 could therefore form the anchoring filaments seen at the basal end of the tip-link complex, and protocadherin-15-CD1 could contribute to the additional strands seen its distal end. Cadherin-23 has been proposed to be a candidate for the tip-link filament (Siemens et al., 2004), and it is possible that cadherin-23 and protocadherin-15 both contribute to the structure of the tiplink complex. Although the ectodomain of cadherin-23 recognized by antibody N1 (Michel et al., 2005) and that of protocadherin-15 recognized by antibody HL5614 are, unlike tip links and transduction (Goodyear and Richardson, 1999; Michel et al., 2005), both subtilisin sensitive, their association with other proteins or as yet unidentified posttranslational modifications may alter the protease sensitivity of molecules that are an integral part of the tip-link complex.

In summary, the results of this study demonstrate the TLA, a protein associated with the tip-link complex of sensory hair cells, is protocadherin-15. Additional studies will now be required to determine whether protocadherin-15 is an integral or peripheral component of this structure and whether it interacts either directly or indirectly with any of the other components of the tiplink complex.

\section{References}

Adato A, Michel V, Kikkawa Y, Reiners J, Alagramam KN, Weil D, Yonekawa $\mathrm{H}$, Wolfrum U, El-Amraoui A, Petit C (2005) Interactions in the network of Usher syndrome type 1 proteins. Hum Mol Genet 14:347-356.

Ahmed ZM, Riazuddin S, Bernstein SL, Ahmed Z, Khan S, Griffith AJ, Morell RJ, Friedman TB, Wilcox ER (2001) Mutations of the protocadherin gene PCDH15 cause Usher syndrome type 1F. Am J Hum Genet 69:25-34.

Ahmed ZM, Riazuddin S, Ahmad J, Bernstein SL, Guo Y, Sabar MF, Sieving P, Griffith AJ, Friedman TB, Belyantseva IA, Wilcox ER (2003) PCDH15 is expressed in the neurosensory epithelium of the eye and ear and mutant alleles are responsible for both USH1F and DFNB23. Hum Mol Genet 12:3215-3223.

Alagramam KN, Murcia CL, Kwon HY, Pawlowski KS, Wright CG, Woychik RP (2001a) The mouse Ames waltzer hearing-loss mutant is caused by mutation of Pcdh15, a novel protocadherin gene. Nat Genet 27:99-102.

Alagramam KN, Yuan H, Kuehn MH, Murcia CL, Wayne S, Srisailpathy CR, Lowry RB, Knaus R, Van Laer L, Bernier FP, Scharwtz S, Lee C, Morton CC, Mullins RF, Ramesh A, Van Camp G, Hageman GS, Woychik RP, Smith RJ (2001b) Mutations in the novel protocadherin PCDH15 cause Usher syndrome type 1F. Hum Mol Genet 10:1709-1718.

Alagramam KN, Stahl JS, Jones SM, Pawlowski KS, Wright CG (2005) Characterization of vestibular dysfunction in the mouse model for Usher syndrome 1F. J Assoc Res Otolaryngol 6:106-118.

Assad JA, Shepherd GM, Corey DP (1991) Tip-link integrity and mechanical transduction in vertebrate hair cells. Neuron 7:985-994.

Bartolami S, Goodyear R, Richardson G (1991) Appearance and distribution of the $275 \mathrm{kD}$ hair-cell antigen during development of the avian inner ear. J Comp Neurol 314:777-788.

Belyantseva IA, Boger ET, Naz S, Frolenkov GI, Sellers JR, Ahmed ZM, Griffith AJ, Friedman TB (2005) Myosin-XVa is required for tip localization of whirlin and differential elongation of hair-cell stereocilia. Nat Cell Biol $7: 148-156$.

Ben-Yosef T, Ness SL, Madeo AC, Bar-Lev A, Wolfman JH, Ahmed ZM, Desnick RJ, Willner JP, Avraham KB, Ostrer H, Oddoux C, Griffith AJ, Friedman TB (2003) A mutation of PCDH15 among Ashkenazi Jews with the type 1 Usher syndrome. N Engl J Med 348:1664-1670.

Boggon TJ, Murray J, Chappuis-Flament S, Wong E, Gumbiner BM, Shapiro L (2002) C-cadherin ectodomain structure and implications for cell adhesion mechanisms. Science 296:1308-1313.

Gale JE, Marcotti W, Kennedy HJ, Kros CJ, Richardson GP (2001) FM1-43 dye behaves as a permeant blocker of the hair-cell mechanotransducer channel. J Neurosci 21:7013-7025.

Goodyear R, Richardson G (1992) Distribution of the 275 kD hair cell antigen and cell surface specialisations on auditory and vestibular hair bundles in the chicken inner ear. J Comp Neurol 325:243-256.

Goodyear R, Richardson G (1999) The ankle-link antigen: an epitope sensitive to calcium chelation associated with the hair-cell surface and the calycal processes of photoreceptors. J Neurosci 19:3761-3772.

Goodyear RJ, Richardson GP (2003) A novel antigen sensitive to calcium chelation that is associated with the tip links and kinocilial links of sensory hair bundles. J Neurosci 23:4878-4887.

Goodyear RJ, Legan PK, Wright MB, Marcotti W, Oganesian A, Coats SA, Booth CJ, Kros CJ, Seifert RA, Bowen-Pope DF, Richardson GP (2003) A receptor-like inositol lipid phosphatase is required for the maturation of developing cochlear hair bundles. J Neurosci 23:9208-9219.

Goodyear RJ, Marcotti W, Kros CJ, Richardson GP (2005) Development and properties of stereociliary link types in hair cells of the mouse cochlea. J Comp Neurol 485:75-85.

Goodyear RJ, McGee J, Weston MD, Legan PK, Bhattacharya G, Cosgrove D, McMillan R, White PC, Walsh EJ, Richardson GP (2006) Identification of the ankle link antigen as the Usher 2c protein, VLGR1, a protein required for cochlear hair bundle development. Assoc Res Otolaryngol Abstr 29:48.

Kachar B, Parakkal M, Kurc M, Zhao Y, Gillespie PG (2000) Highresolution structure of hair-cell tip links. Proc Natl Acad Sci USA 97:13336-13341.

Kondo K, Sagara H, Hirosawa K, Kaga K, Matsushima S, Mabuchi K, Uchimura K, Watanabe T (2002) Hair cell development in vivo and in vitro: analysis by using monoclonal antibody specific to hair cells in the chick inner ear. J Comp Neurol 445:176-198.

Lagziel A, Ahmed ZM, Schultz JM, Morell RJ, Belyantseva IA, Friedman TB (2005) Spatiotemporal pattern and isoforms of cadherin 23 in wild type 
and waltzer mice during inner ear hair cell development. Dev Biol 280:295-306.

Meyers JR, MacDonald RB, Duggan A, Lenzi D, Standaert DG, Corwin JT, Corey DP (2003) Lighting up the senses: FM1-43 loading of sensory cells through nonselective ion channels. J Neurosci 23:4054-4065.

Michel V, Goodyear RJ, Weil D, Marcotti W, Perfettini I, Wolfrum U, Kros CJ, Richardson GP, Petit C (2005) Cadherin 23 is a component of the transient lateral links in the developing hair bundles of cochlear sensory cells. Dev Biol 280:281-294.

Nicolson T, Rusch A, Friedrich RW, Granato M, Ruppersberg JP, NussleinVolhard C (1998) Genetic analysis of vertebrate sensory hair cell mechanosensation: the zebrafish circler mutants. Neuron 20:271-283.

Pickles JO, Comis SD, Osborne MP (1984) Cross-links between stereocilia in the guinea pig organ of Corti, and their possible relation to sensory transduction. Hear Res 15:103-112.

Reiners J, Marker T, Jurgens K, Reidel B, Wolfrum U (2005) Photoreceptor expression of the Usher syndrome type 1 protein protocadherin 15 (USH1F) and its interaction with the scaffold protein harmonin (USH1C). Mol Vis 11:347-355.

Richardson GP, Bartolami S, Russell IJ (1990) Identification of a 275-kD protein associated with the apical surfaces of sensory hair cells in the avian inner ear. J Cell Biol 110:1055-1066.

Rouget-Quermalet V, Giustiniani J, Marie-Cardine A, Beaud G, Besnard F, Loyaux D, Ferrara P, Leroy K, Shimizu N, Gaulard P, Bensussan A, Schmitt C (2006) Protocadherin 15 (PCDH15): a new secreted isoforms and a potential marker for NK/T cell lymphomas. Oncogene 25:2807-2811.

Russell IJ, Richardson GP (1987) The morphology and physiology of hair cells in organotypic cultures of the mouse cochlea. Hear Res 31:9-24.

Seiler C, Finger-Baier KC, Rinner O, Makhankov YV, Schwarz H, Neuhauss SC, Nicolson T (2005) Duplicated genes with split functions: independent roles of protocadherin 15 orthologues in zebrafish hearing and vision. Development 132:615-623.

Senften M, Schwander M, Kazmierczak P, Lillo C, Shin JB, Hasson T, Geleoc GS, Gillespie PG, Williams D, Holt JR, Muller U (2006) Physical and functional interaction between protocadherin 15 and myosin VIIa in mechanosensory hair cells. J Neurosci 26:2060-2071.

Sheng M, Sala C (2001) PDZ domains and the organization of supramolecular complexes. Annu Rev Neurosci 24:1-29.

Siemens J, Lillo C, Dumont RA, Reynolds A, Williams DS, Gillespie PG, Muller U (2004) Cadherin 23 is a component of the tip link in hair-cell stereocilia. Nature 428:950-955.

Thorpe JR (1999) The application of LR gold resin for immunogold labelling. Methods Mol Biol 117:99-110.

Tsuprun V, Goodyear RJ, Richardson GP (2004) The structure of tip links and kinocilial links in avian sensory hair bundles. Biophys J 87:4106-4112.

Zhao Y, Yamoah EN, Gillespie PG (1996) Regeneration of broken tip links and restoration of mechanical transduction in hair cells. Proc Natl Acad Sci USA 93:15469-15474. 